\title{
A Survey of Measurement Templates Used for Assessing Pre-Operative Body Contours and Evaluating Cosmetic Surgery Results Using a 3D Whole-Body Scanner
}

\author{
David B. STEFAN ${ }^{1}$, David A. GILBERT ${ }^{2}$ \\ ${ }^{1}$ Novaptus Systems Inc., Chesapeake VA, USA; \\ ${ }^{2}$ The Hague Plastic and Cosmetic Surgery Center, Norfolk VA, USA \\ DOI: $10.15221 / 17.239$ http://dx.doi.org/10.15221/17.239
}

\begin{abstract}
Background

A 3D whole-body scanner has been utilized at a cosmetic surgery practice in Norfolk, Virginia since 2002. Pre-operative and post-operative scans have been taken of many patients that have undergone various cosmetic surgical procedures. Through trial, error and analysis, various measurement templates have been designed as an aid for the surgeon to assess the pre-operative body contours and to evaluate the post-operative results of common cosmetic procedures. This paper presents measurement templates that have been found to be useful for breast augmentation, breast reduction, abdominoplasty and thigh-lift surgical procedures.
\end{abstract}

\begin{abstract}
Methods
Manual measurements have traditionally been used to evaluate the body contours of the pre-operative patient. These are typically of linear and circumferential nature. Many of the manual measurements made by the surgeon are replicated within the scanner measurement software. These can be selected and programmed into a measurement template, which can then be applied to the patient's 3D body model to automatically extract the desired information. The scanner measurement software can produce multidimensional measurement information such as surface area and volume. Such measurement tools were previously unavailable. There are many measurements provided by scanning software that are beneficial to enhance the pre-operative evaluation, perhaps too many. These include linear contours, the height of certain measurements and uncommon circumferential measurements. The measurement templates presented are a result of experience. Case 1 and Case 2 detail the measurement template used for evaluating breast augmentation and breast reduction procedures. Case 3 is an example of the measurement template used for evaluating an abdominoplasty procedure. The measurements utilized for abdominoplasty center around the mid-section of the body. Case 4 is an example of the template used for evaluating a thigh-lift. The measurements utilized are focused on the lower half of the body.
\end{abstract}

\section{Discussion}

Applying procedure-specific measurements to a pre-operative 3D body scan can help detect and quantify existing asymmetries. These asymmetries may be corrected during the surgical procedure through proper planning. Breast augmentation, reduction, abdominoplasty and thigh-lift surgical procedures have immediate impact on the body. These changes can be measured longitudinally as patients return for post-operative visits. The effects of edema (swelling) can be documented with each post-operative 3D body scan. The edema usually abates within six months and the 3D body scans thereafter can be compared with the pre-operative 3D body scan to quantify the physical changes brought about by the surgical procedure. Changes in volume can be reviewed against the weight of the actual amount of tissue removed in cases of breast reduction, thigh lift, or abdominoplasty. In the case of breast augmentation, changes in post-operative bust volume can be compared to the size of the breast implants inserted.

\section{Conclusion}

The measurement templates used to document the cosmetic surgical procedures discussed have evolved over time. They are in no way final or complete, but they have added considerable multidimensional insight into evaluating pre-operative body contours and documenting any pre-existing asymmetric conditions. Applying these templates to post-operative 3D body scans quantifies the physical changes brought by the surgical procedure, including the correction of detected asymmetries. Moreover, the series of pre-operative and post-operative 3D body scans form the patient's 3D digital archive. As new measurements are added to the existing measurement templates, they can be applied to 3D body models of past surgical procedures for investigation and validation. 


\section{Introduction - 3D body scanner history and evolution}

IRB NUMBER 05-09-FB-0235 Eastern Virginia Medical School provides the basis for this research and presentation, to which the subjects have signed the appropriate consent forms.

November of 2002 was the month that a 3D whole-body scanner was installed at The Hague Center for Cosmetic Surgery, in Norfolk, Virginia, USA. It was ordered in September of that year. The serial number assigned to the scanning system was 005 , making it the fifth 3D whole-body scanner from the vendor, who began shipping this configuration earlier in that year.

The fully assembled system spanned 16 feet in length, 8 feet in width, 7 feet in height and was shaped like a diamond that is found on a suite of playing cards. A dark, heavy cloth, fastened by Velcro wrapped around the entire frame. Aluminum support beams overhead were used to give the frame structural integrity. A large cloth covering was draped over these beams.

The entrance to the scanner was a curtain-like crease. The person to be scanned would enter the scan chamber, and the operator would close this opening by pressing the Velcro seams together. Every possible effort was made to ensure that no external light would enter the scan chamber during scan acquisition.

The scan acquisition equipment consisted of four "sensor heads," two per each end of the scanner and stacked one on top of the other in a metal frame. Each sensor head contained a white-light projector mechanism and a camera with a finely ground lens and mechanical aperture. During the acquisition process, each sensor would travel a pre-programmed distance along the y-axis, stopping at a predetermined height. The projectors would stop in front of one of two metal grids, each with a specific pattern. The projector light would activate, and a horizontal pattern of light and dark would be cast upon the subject in the scan chamber.

The mechanical camera aperture would open and shut, acquiring the reflection of the light projected on the subject. Each camera would capture eight successive images on this first pass, for a total of 32 images.

The sensor heads would return to their original positions and begin the second pass. At the appropriate elevation, a different grid would be presented in front of the projector light. This grid had finer lines than the grid employed in the first acquisition pass. Once again, the mechanical apertures of the cameras would open and shut in noisy succession. Each camera captured another eight images. This would result in a collection of 32 additional images having a different structured light reflection than those images collected during the first pass.

The 64 total images would be gathered and stored by the system computer. The time required for raw image acquisition was nearly 60 seconds. To further date the technology employed back in 2002, the system computer was running the Windows NT operating system and did not come with a networking port, only a dial-up modem.

The software program would assemble these raw images, taken from different elevations and with different structured light patterns and combine them in such a way as to make a large whole-body 3D binary file. This file was too large to manipulate and measure, so it was decimated into a smaller file, known as a "reduced binary data" file (rbd).

This rbd file was constructed in a manner that the measurement software could search for known landmarks on the body surface such as the "small of the back," the "back of the neck," and find the separation points between the legs and the arms from the torso.

Once these and other landmarks are located, measurements could be extracted from the 3D body model. The measurements extracted depended on the measurements programmed into a "measurement extraction profile." Hundreds of measurements are available. These range from common circumferential measurements, heights (from the floor) of those measurements, linear measurements, surface area measurements and volume measurements.

This early, "booth-type" 3D whole body scanner used a technique called "white light phase profilometry" to acquire images and create a 3D body model. Its primary usage was within the clothing industry, either for custom tailored clothing or for clothes sizing studies based on a cross-section of a population. The measurement extraction profiles supplied by the system software were measurements for creating custom-tailored garments. Most of these profiles did not have a medical analog. 
The time from the start of the scan acquisition process to the presentation of the $3 \mathrm{D}$ body model on the computer display took nearly 3 minutes. It was then that the 3D image could be evaluated by the operator. This would be the moment that the operator could decide to accept the 3D body image, or notice errors in the 3D body model construction that would require the subject to be re-scanned. Typical errors would include posture problems, inappropriate garments (dark undergarments that absorbed the light), or the wearing of jewelry that would scatter the projected light resulting in minor image distortion, etc. These incidents could be minimized by proper training. However, if there was outside light entering the scan chamber, or movement of the individual during the scan acquisition process, it could affect the image construction in such a way as to distort entire areas of the body. This would render the scan useless.

This is a description of the starting environment in which we began applying what was then the "latest and most up-to-date" 3D body scanning technology available, attempting to measure and evaluate the body contours of pre-operative cosmetic surgery patients.

\section{The premise for utilizing a 3D body scanner in the cosmetic surgery clinic}

Cosmetic surgery is as much art as it is science. A thorough understanding of the patient's pre-operative body contours is essential to planning the surgical procedure. By employing the use of the 3D body scanner to acquire an accurate, measurable 3D body model of the patient prior to surgery, and programming and applying surgery-specific measurement templates to this $3 \mathrm{D}$ body model, the extracted measurements might provide multidimensional insight - a new way - of evaluating body contours. Moreover, since the 3D body model could be archived, the patient could be scanned postoperatively as part of routine follow-up visits. The same measurement template that was applied to the pre-operative 3D body model could be applied to the post-operative 3D body model. Measurements could be extracted and compared. The results of the surgery could be validated. The entire series of pre- and post-operative scans would be placed in the patient's 3D digital archive and complement his or her conventional medical record.

\section{Examination of 3D body scanner as an aid for "common" cosmetic surgery cases}

\section{Case 1. Breast Augmentation}

Breast augmentation can be considered a common cosmetic surgery procedure, and yet every case is unique. Often, patients arrive with a preconceived notion of their desired post-operative appearance. This appearance may or may not be surgically achievable. The surgeon's primary obligation is to evaluate the pre-operative physical state of the body and investigate any background medical condition that might jeopardize the patient's health, then decide whether to perform the desired surgery or not.

If the surgeon feels confident that a successful outcome is probable, the patient's age, physical condition and overall shape of their body are considered. A patient that has muscle tone and definition is, from a surgical standpoint, treated in a different manner than a patient that has skin laxity. Even the patient's posture is taken into consideration.

Figure 1 depicts a series of illustrations that outline the breast augmentation procedure.
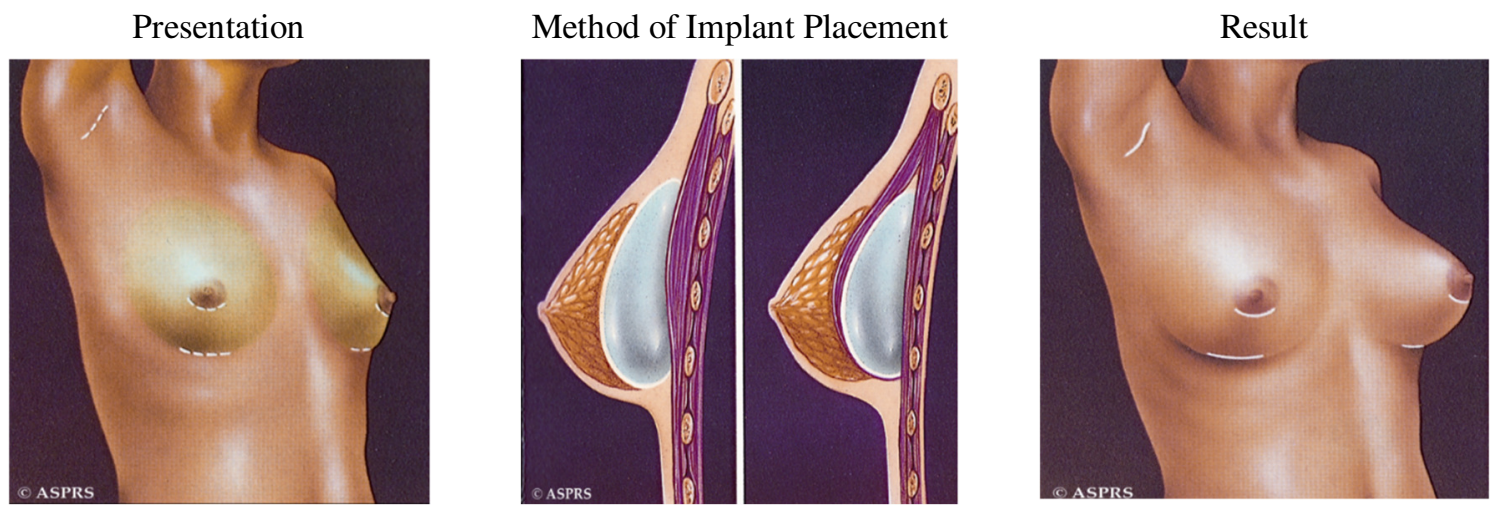

Figure 1. Artist's Rendering of Breast Augmentation Procedure 
The dotted lines on the leftmost figure indicate possible areas of incision for implant insertion. The implants could be positioned behind the breast tissue, or behind the muscle of the chest wall. Depending on the surgical approach, scarring of the incision areas will result, as indicated in the rightmost image.

The primary focus for pre-operative evaluation is the present shape and contours of the patient's breasts. This varies greatly from individual to individual.

There are two conditions of interest to the surgeon. The first is the detection of any existing breast asymmetries. The second is to determine whether the breasts are ptotic, and if so, to what degree.

If the surgeon does not accurately evaluate pre-operative breast asymmetries and proceeds with the breast augmentation procedure, the outcome is usually an amplification of any existing asymmetry, exacerbated by the volume of the breast implant.

Detecting breast asymmetry is not necessarily a straightforward process. The overall shape of the breasts are determined by volume, contours along the chest wall and surface of the breasts, as well as circumferential and linear measurements. One often hears the term "fullness," which is considered a "quality" that is hard to quantify. Unless the asymmetry is obvious, mere visual observation can often be misleading. One helpful technique is to take a $2 \mathrm{D}$ frontal photograph of the pre-operative breasts and turn the photograph upside down. This has the effect of removing from the mind a common viewing construct, allowing one to identify differences in the shape of the breasts, if any, more clearly.

Figure 2 displays a patient with some breast asymmetry. The photograph on the right has been rotated 180 degrees, making the asymmetry appear more pronounced and identifiable.
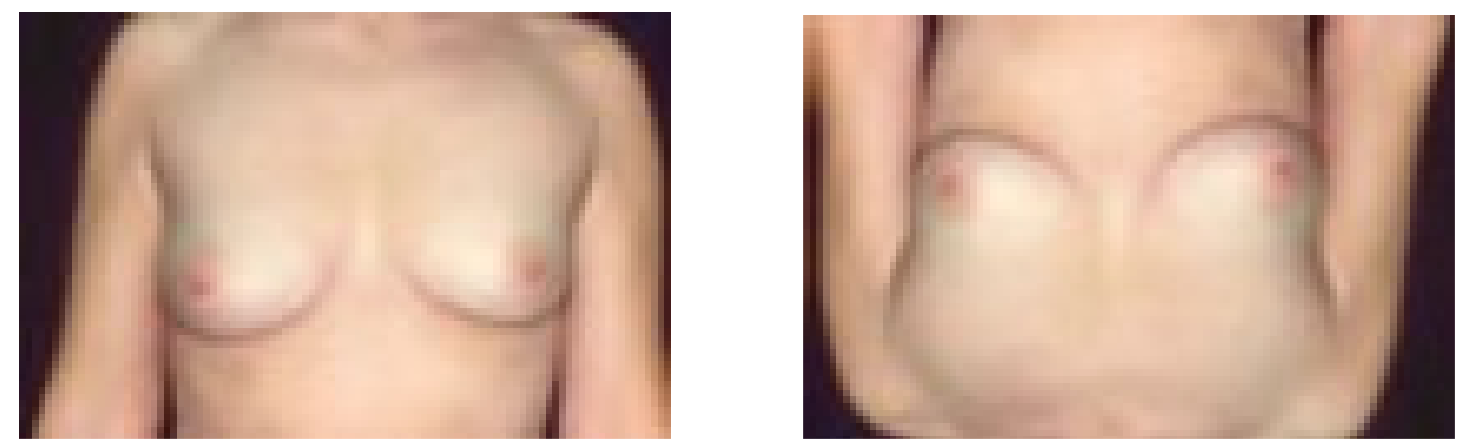

Figure 2. One Technique to Assist Determination of Pre-Operative Breast Asymmetries

Ptosis is a pathological term defined as a prolapse or "drooping" of any organ. For the breasts, this is when the nipples begin to point downward instead of being angled slightly higher or perpendicular to the floor. The breasts begin to lose their "fullness" of shape, and have a flaccid, pendulous appearance. Age plays a role in this situation, but it also a common aftereffect of pregnancy and nursing, particularly after multiple pregnancies.

A ptotic breast condition is rarely correctable by a diet or exercise regime, but it can be mitigated to a certain extent by arresting the progression.

Thus, the ptotic condition must be evaluated by the degree of the ptosis. Patients with extremely ptotic breasts are not candidates for direct breast implants. In many cases, the ptotic breasts must be reshaped, and the nipple and areola complex relocated during the augmentation surgery to accommodate the implant and obtain the desired appearance. This procedure is known as a mastopexy, or a fixation of a pendulous breast, and is similar to the breast reduction procedure discussed below.

Figure 3 depicts a patient who presents with a ptotic breast condition. In this situation, a mastopexy procedure appears to be the appropriate path. 

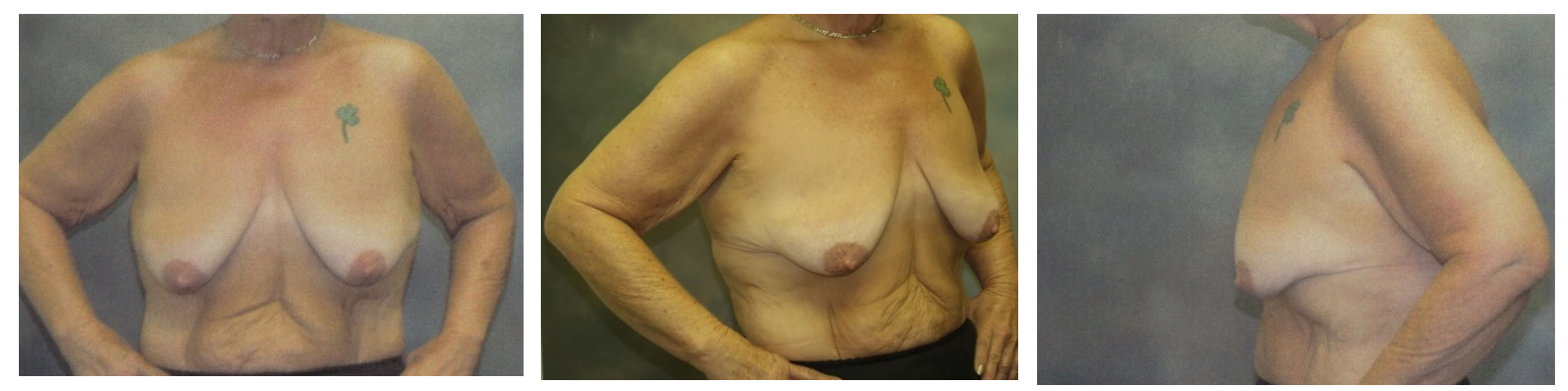

Figure 3. An Example of a Ptotic Breast Condition

\section{Traditional breast measurements - The clothing world vs. The medical world}

The difference between the image in the mind of the patient for her desired post-surgical appearance prior to the breast augmentation procedure and the surgeon's capabilities to achieve the patient's wishes can often be wide. Both have the same general idea of the desired outcome in terms of aesthetic appearance, yet they cannot speak to each other in the same language.

The patient has been accustomed to think in terms of clothing sizes. These include circumferential bust, waist and hips measurements. "34-24-34," is a term from "the old days" that conjures up an image of a woman with a shapely, hourglass figure and fulsome breasts. "Petite" suggests a smaller stature. Size 2 and Size 4 can be associated with thin appearance. To the patient, the bra size implies the breast size, with the volume of the breasts designated by the size of the "cup." "34A" and "34D" refer more to the volume of the breasts than to the bust circumference.

A female patient who desires a breast augmentation procedure might presuppose that the surgeon understands her desire to be "two cup sizes larger."

This is not how the surgeon has been trained. The surgeon thinks in terms of implant volume in cubic centimeters (cc), and whether the implant should be saline or silicone, with a high, medium or low profile, and whether the implants should be positioned behind the breast tissue or behind the chest wall muscle.

This inability for both parties involved to "concur" on the same post-operative appearance prior to the procedure sometimes leads to disappointment on the patient's part after the surgery has been performed, the swelling has abated and the breast implants have settled. The surgeon may have the opinion that the results are superb. The breasts appear symmetrical, the nipple complex, though they may have been relocated are in the proper position, and the reconstructed areolas are each of the same size and shape. Scarring has been minimized, too. From the surgical viewpoint, the procedure was an "out and out" success.

Yet the final judge in this case is the patient. Somehow, somewhere, the image the patient had in mind for the surgical results differed enough from the surgeon's image that mild discontentment sets in.

"Doctor, I like my new breasts...but," is a comment that can sometimes be heard. If the displeasure persists, a "redo" of the surgical procedure may occur. Many situations like this could have been avoided if only the patient and the surgeon "spoke" a common language, or if, somehow, a translation of the language of the clothing world to that of the medical world was possible.

This is where the idea of employing a 3D body scanner can add value. 3D body scanning has a robust background in the garment industry, from extensive population studies for standardizing clothing sizes and mannequin production, to measuring individuals for constructing custom-fit garments. The measuring software to extract measurements from the 3D body model is programmable, and custom templates might be developed that would produce measurement information meaningful to the surgeon. The existing measurement templates provided with the scanner software for clothing garments would produce measurement information that the patient could understand.

Thus, the extensive measuring capabilities of the scanning software could serve two functions using the same 3D body scan. Measurements could be utilized that could extract information that the patient is familiar with, such as bust circumference and bra cup size. Separately, measurements could be programmed that could extract information such as linear and contoured distances from the suprasternal notch to the left and right nipple, bust prominences, and, if accurate enough, even the volume of each breast. These would be of interest to the surgeon. 
Moreover, measurements could be extracted from the entire 3D body model, allowing both the surgeon and the patient to understand her existing proportions by using the waist circumference, hips circumference, etc.

The question then arises as to how to construct the appropriate measurement profile. Which measurements are required to replicate measurements manually made by the surgeon? What measurements can be added that can only be produced by the 3D measurement software that might be able to add unique insight into the pre-operative evaluation?

Figure 4 depicts 2D photographs of a pre-operative breast augmentation patient. The images underneath are screen captures of the equivalent area of the breasts from the patient's pre-operative $3 \mathrm{D}$ body model. Already we see a difference in presentation that the surgeon may not be familiar with.
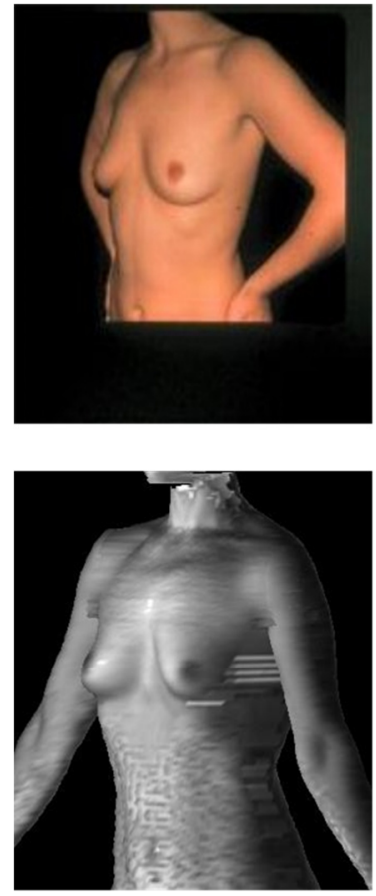

Left Side Close-up
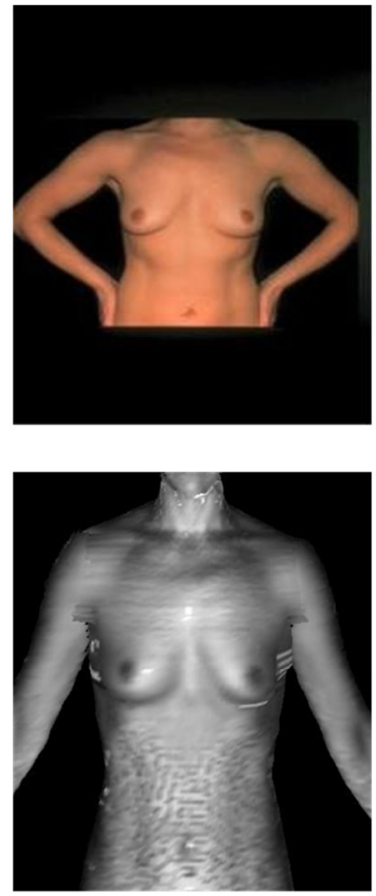

Center Close-up
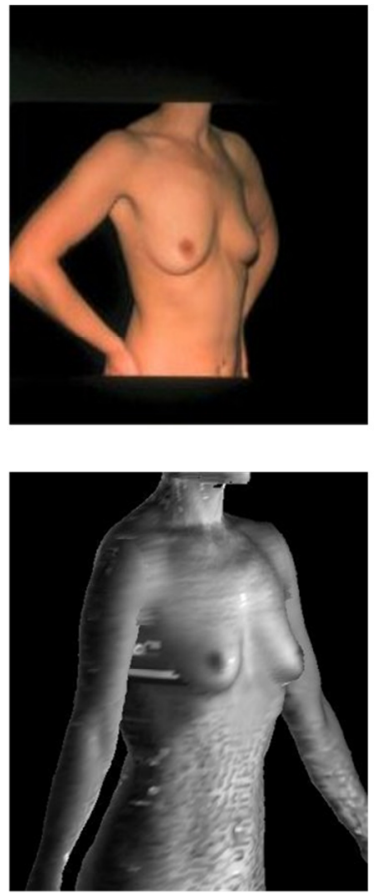

Right Side Close-up

Figure 4. 2D Photographs and 3D Body Model Images of the Same Patient

The series of photographs above were taken by the surgeon as a normal part of documenting the preoperative breast contours. These were taken from different angles and different length; hence each view of the patient has a different perspective distance. The bottom images were captured by rotating the $3 \mathrm{D}$ body model. Perspective and distance are maintained.

The patient is a female in her mid-twenties, and her breasts appear to have a minor ptotic condition. The chest area below her neck and shoulders above the breasts is thin, reflective of the rest of her body.

A tape measure is then utilized. The patient stands at normal posture. The surgeon measures the distance from her suprasternal notch, along the contours of the chest and breast to the left and right nipple complex. These numbers are recorded. Another measurement is from the distance between the nipples, parallel to the floor. Her bust circumference is also noted.

It is at this point that the surgeon then begins to consider the appropriate size of the implant, as well as the type of implant. The wishes of the patient are then taken into consideration.

The pre-operative breast augmentation evaluation used linear measurements, contoured or straight, circumferential measurements and, by way of selecting breast implants, volume considerations. 


\section{D body scanning to measure breast contours}

The 3D body scanner measurement software can generate hundreds of measurements from the 3D body model. The number of measurements that can be programmed for the upper body and the chest and bust area are numerous. It is quite possible to "over-measure" the chest and bust area, and produce too much measurement data.

Once the 3D body model has been produced, the selected measurement profile automatically extracts and displays measurements. These measurements are based on the body landmarks that are identified by the measurement software during the creation of the 3D body model. It was found that the automatic location of certain landmarks, particularly associated with the chest and bust areas were not necessarily in agreement with the surgeon's opinion. Many of these landmarks and the endpoints that determine the distance between measurements can be manually adjusted to accord with the surgeon's judgement. Once manually adjusted, the measurement template is reapplied to the $3 \mathrm{D}$ body model and the extracted measurements are presented.

A survey of the available upper body, chest and bust-specific measurements found that several available measures could be programmed to replicate the surgeon's manual measurement efforts.

The first measurement profile was constructed by selecting measurements thought to be useful for detecting the existence of breast asymmetry. One of the landmarks that the measurement software looks for on the 3D body model as a reference point is the suprasternal notch. The measurement nomenclature used by the scanner software is "Neck_Front_Center."

The measurements "FrontNeck2Bust_Left" and "FrontNeck2Bust_Right" compare to the manual measurements made by the surgeon to determine the symmetry of the nipple complex. The "Bust_Promenance_Left" and "Bust_Prominance_Right" measure the lateral contour of each breast. The measurement "Bust2Bust_Horizontal" measures the linear distance between the nipple complex of each breast.

The application of these measurements to the pre-operative 3D body model result in a triangle pattern when the measurement extraction lines are displayed. A quick glance at the symmetry of this triangle gives clues to any asymmetries that may exist between the breasts.

Gradually, measurements associated with the chest area, upper body and bust were added to a measurement extraction profile created for evaluating pre-operative breast augmentation (and breast reduction) patients. The measurement profile would also be useful to evaluate measurement changes post-operatively, validating the results of the surgery.

Figure 5 depicts this measurement profile applied to the patient's 3D body model prior to breast augmentation surgery. Note the "near" symmetrical triangle formed between the patient's suprasternal notch and nipple complexes. The line descending from the suprasternal notch terminates at the "waist front" point, which is the red dot above the abdomen. The yellow line below the horizontal line between the breasts is not displayed. The "waist front" point is not an adjustable point.

The measurements extracted indicate a 2-mm length difference from the suprasternal notch to the left nipple compared to the suprasternal notch to the right nipple (FrontNeck2Bust). The "Bust_Prominance" measures $5 \mathrm{~mm}$ larger for the right breast. These are within the scanner measurement tolerances per the calibration process. Thus, the pre-operative patient is considered to have symmetrical breasts.

The patient was scanned two months after surgery. The same measurement template was applied.

Figure 6 presents the results of the measurement template applied to the patient's 3D body model acquired two months after the breast augmentation procedure.

Measurements concerning post-operative asymmetries, such as Bust_Prominance and FrontNeck2Bust appear consistent with the appearance of a symmetrical surgery result. 

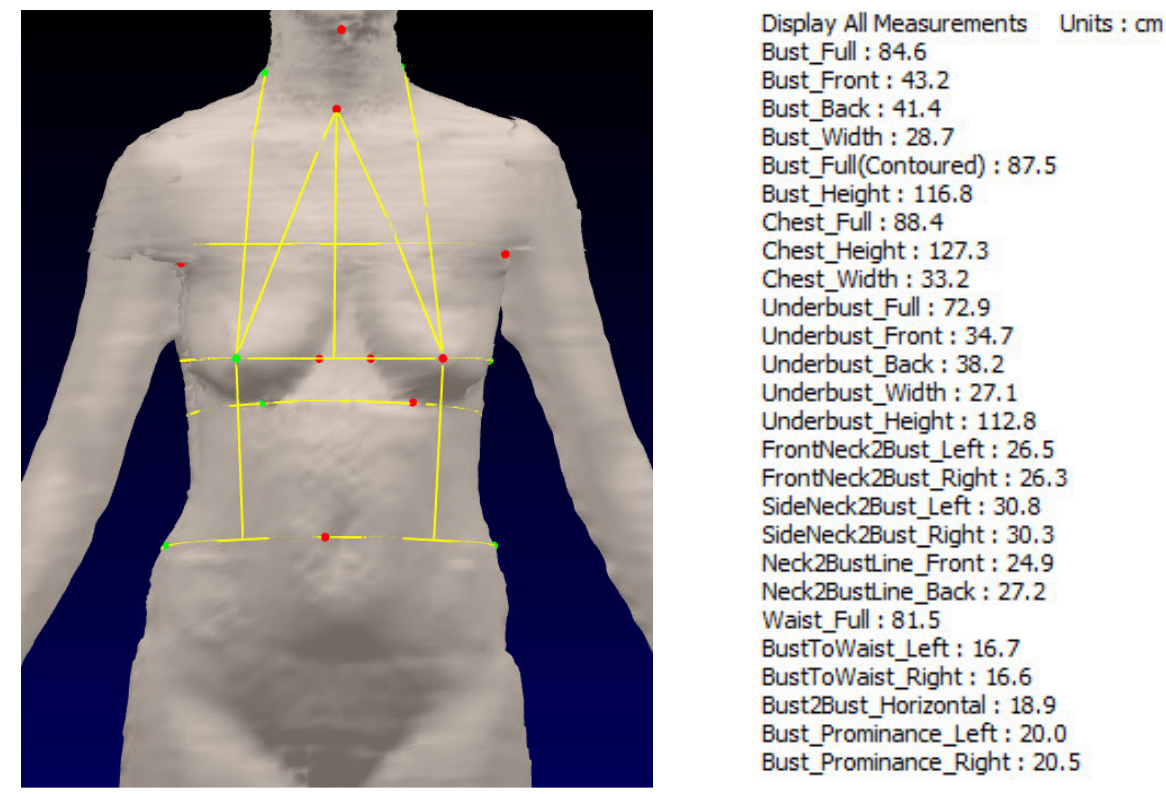

Figure 5. Breast Measurement Template Applied to Pre-Operative 3D Body Model
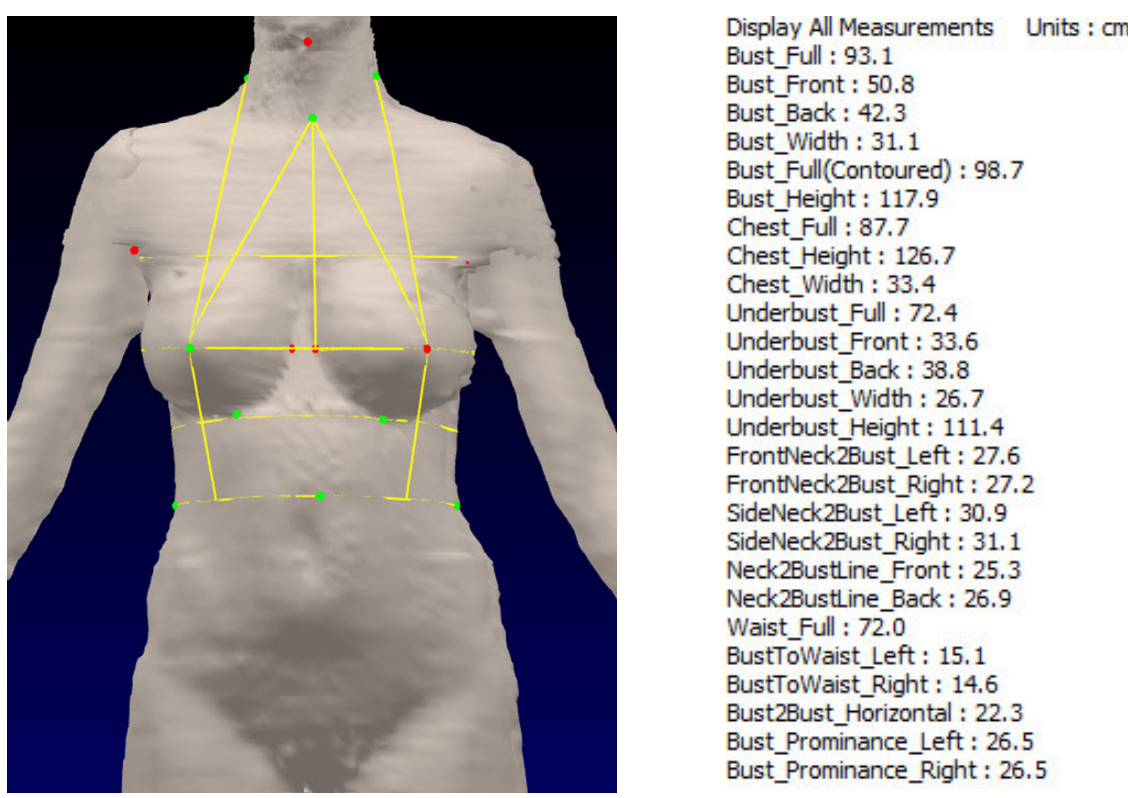

Figure 6. Breast Measurement Template Applied to the Post-Operative 3D Body Model

Figure 7 presents the differences between the pre-operative and 2-month post-operative measurements. 


\author{
Display All Measurements Units : $\mathrm{cm}$ \\ Bust_Full : 84.6 \\ Bust_Front : 43.2 \\ Bust_Back: 41.4 \\ Bust_Width : 28.7 \\ Bust_Full(Contoured) $: 87.5$ \\ Bust_Height : 116.8 \\ Chest_Full : 88.4 \\ Chest_Height : 127.3 \\ Chest_Width : 33.2 \\ Underbust Full : 72.9 \\ Underbust_Front : 34.7 \\ Underbust Back : 38.2 \\ Underbust_Width : 27.1 \\ Underbust Height : 112.8 \\ FrontNeck2Bust_Left : 26.5 \\ FrontNeck2Bust_Right : 26.3 \\ SideNeck2Bust_Left : 30.8 \\ SideNeck2Bust Right : 30.3 \\ Neck2Bustline_Front: 24.9 \\ Neck2Bustline_Back : 27.2 \\ Waist_Full : 81.5 \\ BustToWaist_Left : 16.7 \\ BustToWaist_Right : 16.6 \\ Bust2Bust Horizontal : 18.9 \\ Bust_Prominance_Left : 20.0 \\ Bust_Prominance_Right : 20.5
}

Pre-Op Extracted Measurements

\author{
Display All Measurements Units : cm \\ Bust_Full : 93.1 \\ Bust Front : 50.8 \\ Bust_Back: 42.3 \\ Bust Width : 31.1 \\ Bust_Full(Contoured) : 98.7 \\ Bust Height : 117.9 \\ Chest_Full : 87.7 \\ Chest_Height : 126.7 \\ Chest_Width : 33.4 \\ Underbust_Full : 72.4 \\ Underbust_Front : 33.6 \\ Underbust_Back : 38.8 \\ Underbust_Width : 26.7 \\ Underbust_Height : 111.4 \\ FrontNeck2Bust_Left : 27.6 \\ FrontNeck2Bust_Right : 27.2 \\ SideNeck2Bust_Left : 30.9 \\ SideNeck2Bust Right : 31.1 \\ Neck2BustLine_Front : 25.3 \\ Neck2Bustline_Back: 26.9 \\ Waist_Full : 72.0 \\ BustToWaist_Left : 15.1 \\ BustToWaist_Right : 14.6 \\ Bust2Bust_Horizontal : 22.3 \\ Bust_Prominance_Left : 26.5 \\ Bust_Prominance_Right : 26.5
}

2-Month Post-Op Extracted Measurements

Figure 7. Comparison of Pre-Operative and Post-Operative Breast Augmentation Measurements

The scanner software's capabilities for measuring linear, contoured linear and circumferential measurements appear helpful in assisting the surgeon to identify and evaluate pre-operative breast asymmetries. The software is also of value in validating the correction of these asymmetries postsurgery, as indicated by the "Bust_Prominance" and "FrontNeck2Bust" measurement values.

Figure 8 display a pre-operative photograph of a breast augmentation patient that has noticeable breast asymmetry. The image to the right of the photograph is a similar view as presented by her 3D body model produced by the 3D scanner.

Figure 9 depicts the result of applying the breast measurement template to this 3D body model, and clearly indicates that the right breast is substantially larger than the left breast. Note that the extracted measurements indicate that her right bust prominence is $2.6 \mathrm{~cm}$ larger than her left, and the distance from the suprasternal notch to her right nipple complex is $2.6 \mathrm{~cm}$ longer than the distance from the suprasternal notch to her left nipple complex.
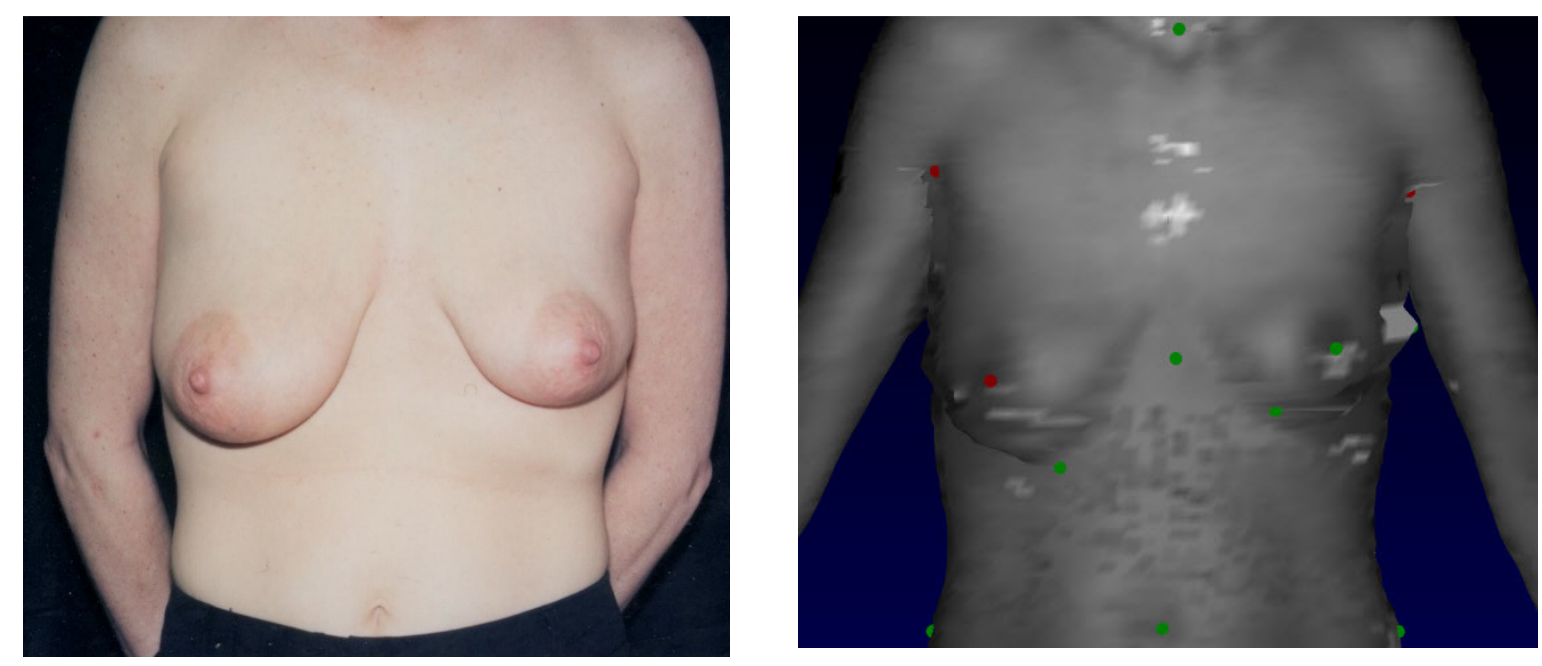

Figure 8. Pre-Operative Photo and Pre-Operative 3D Model Image Comparison 

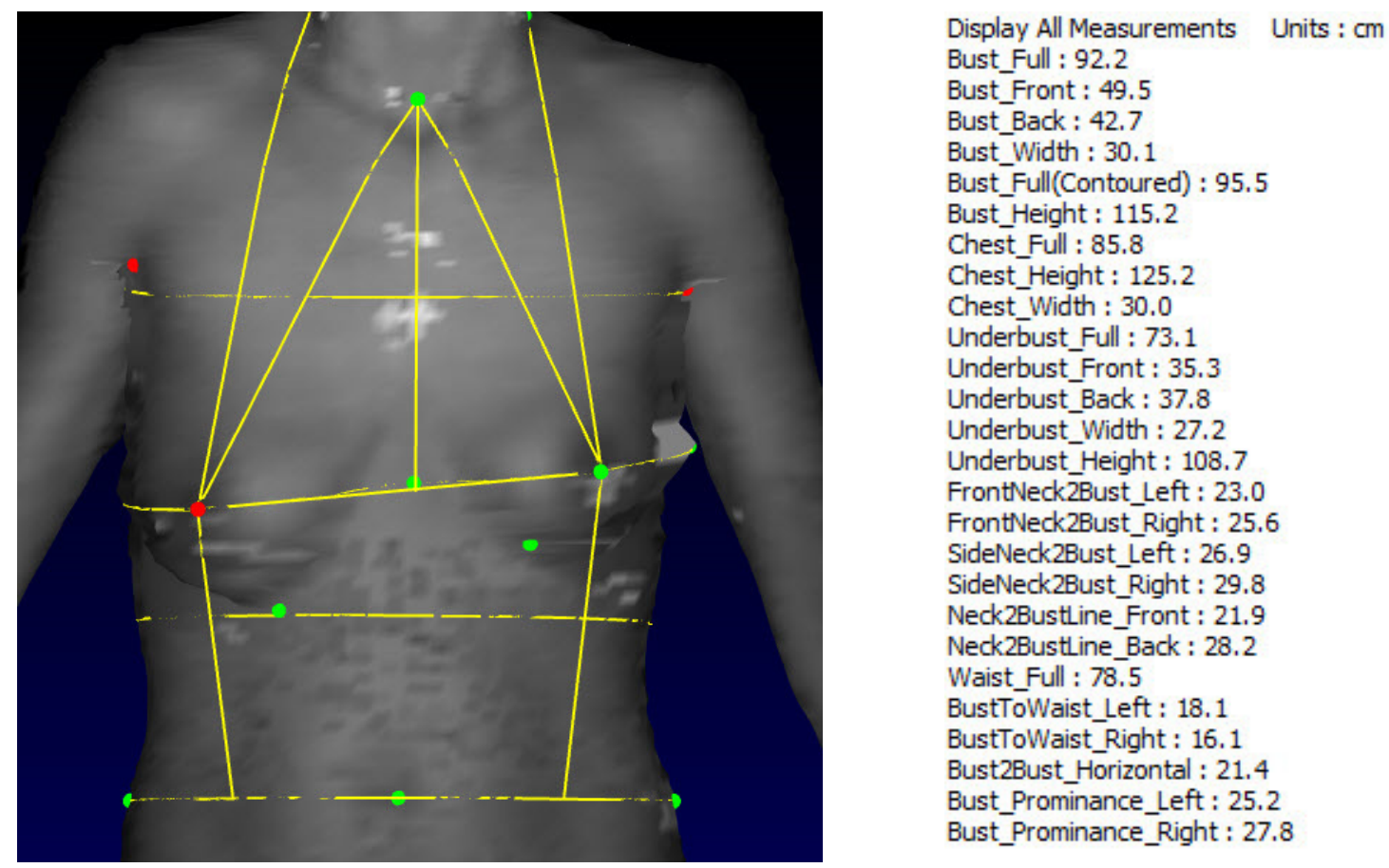

Figure 9. Measurements Extracted from 3D Body Model Indicates Degree of Breast Asymmetry

\section{Bust/breast volume determination using the 3D body scanner and measurement tools}

Here is where we begin to see the difference between how the clothing world measures "the bust," and what the medical world considers "the breast."

The scanner software provided a measurement template to evaluate bust size and bust volume. This measurement profile was designed by the scanner manufacturer to assist clothing undergarment producers for design of brassieres. For illustrative purposes, Figure 10 displays the measurement lines corresponding to application of this template on a patient that underwent a breast augmentation procedure, and a section of the corresponding extracted measurements.
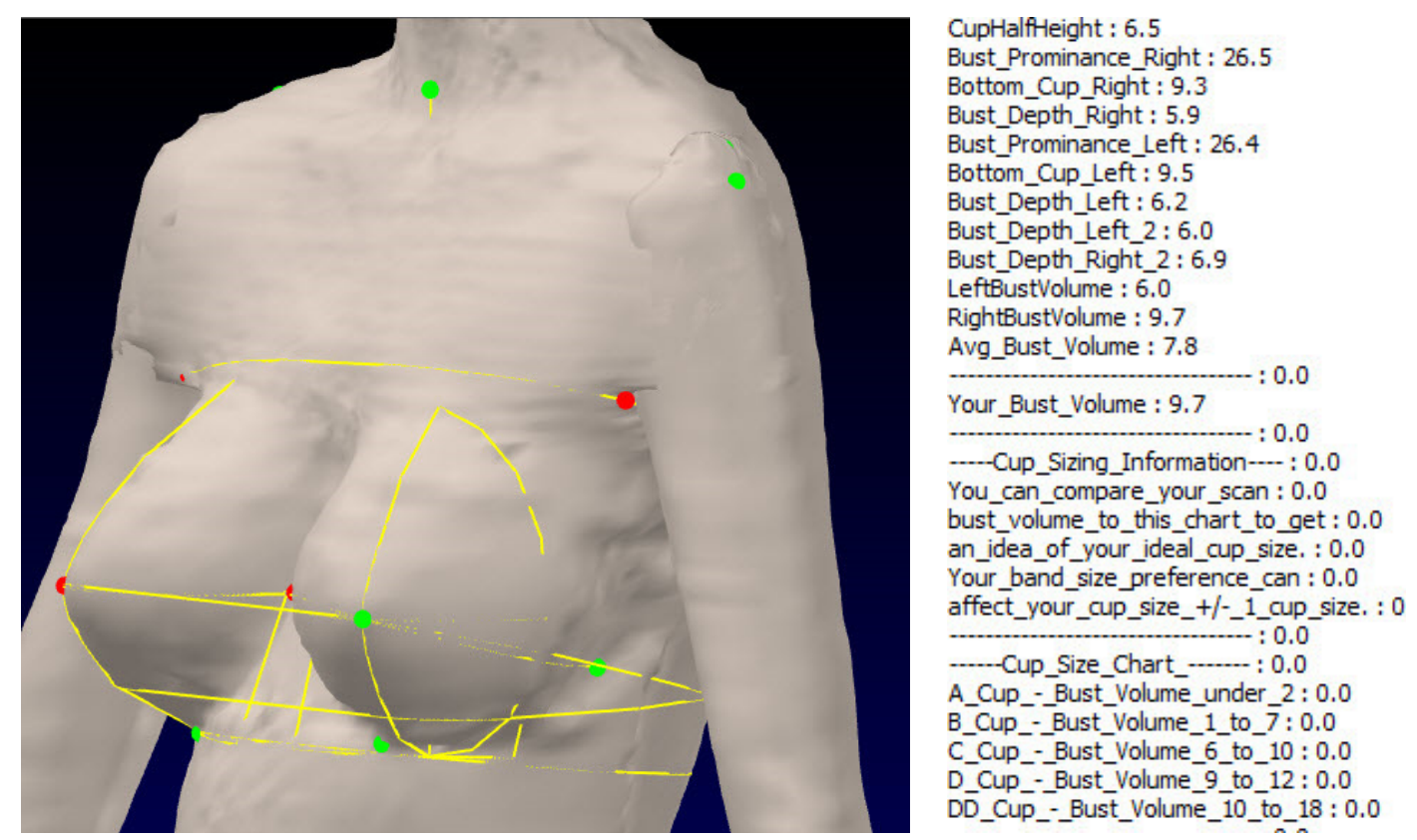

Figure 10. Application of a Bust Measurement Profile for Brassiere Construction 
One can readily observe that the measurements utilized in this profile are not directed towards the medical definition of the breast.

Technically, the breast area can consider to begin at a point known on the chest as the "Upper Medial Pole." This point is near the suprasternal notch. Moving counterclockwise, a contour line can be drawn to a point known as the "Upper Lateral Pole." This is above the armpit and below the deltoid. Together, these points outline the beginning of the curvature of the breasts as they separate from the chest wall.

The outline continues along the axillary fold of the breasts, which are the contours following the outer portions of the breasts. This contour ends at the "Lateral Breast Pole." This is the point where the breasts begin an inward curve.

The "inframammary fold" is the contour underneath the intersection of the breast and the chest. Ptotic breasts can extend downward quite a distance from this fold. Proportional breasts having the quality of "fullness" will have a smaller distance from the lowest point on the breast to the inframammary fold. Small breasts can appear to have no lower breast distance from the inframammary fold, or to have no inframammary fold at all.

The inframammary fold ends at the "Lower Medial Pole," where the breast begins to curve upward. The contour line then traverses the "Medial Fold," and then terminates at the Upper Medial Pole, where we first began to draw the outline of the breast.

Figure 11 depicts the outline of the breast as described above.

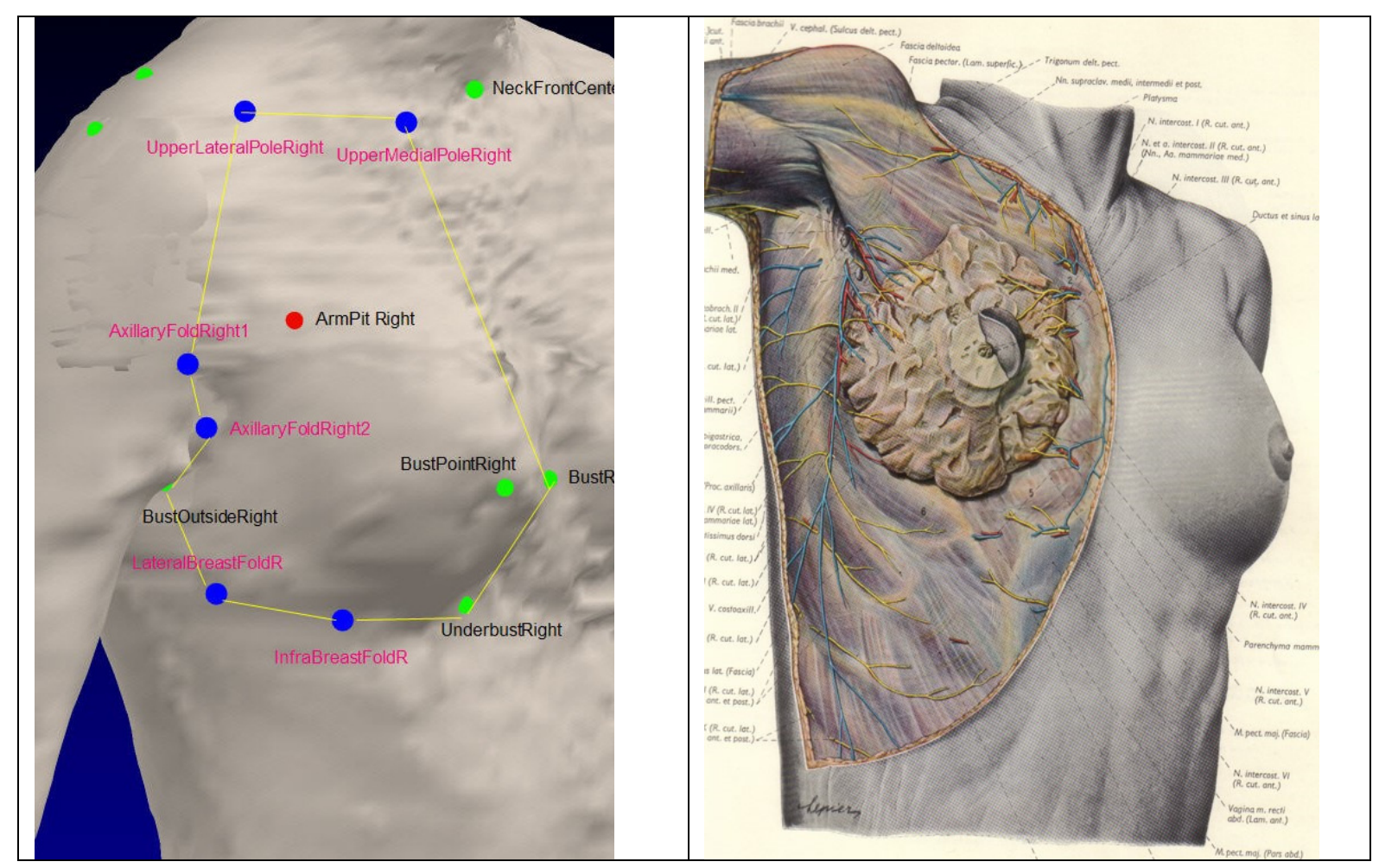

Figure 11. Medical Definition of Outline of a Breast

Observation of the breast folds help to identify asymmetrical and ptotic conditions. It also uncovers areas surrounding the breast that may require an additional contouring procedure during the surgery.

Figure 10 depicted the result of applying a measurement template designed to assist brassiere manufacturers. Note the circular outlines of each "breast." The software is attempting to find the volume of each breast so that a clothing manufacturer can accommodate the cup size, the brassiere straps across the shoulders, and the strap and clasp for the bust circumference

The measurement information provided by this profile is of limited use to the surgeon, who defines the breast area as outlined in Figure 11. 
To help our investigation, the scanner manufacturer provided a programmable measurement template based upon a specification written by the surgeon. This measurement profile contained movable points along the breast area, as well as an adjustable "depth" point below the nipple location.

Figure 12 displays the measurement lines associated with this "breast volume" measurement profile. 3D body models are presented in color, surface and point cloud displays. The surgeon has adjusted each movable point associated with this measurement profile to their appropriate positions.

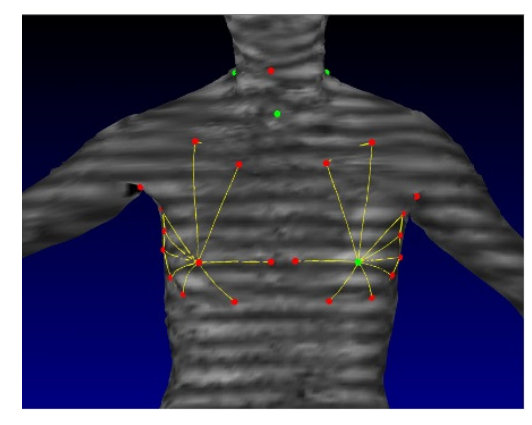

"Color" Mode

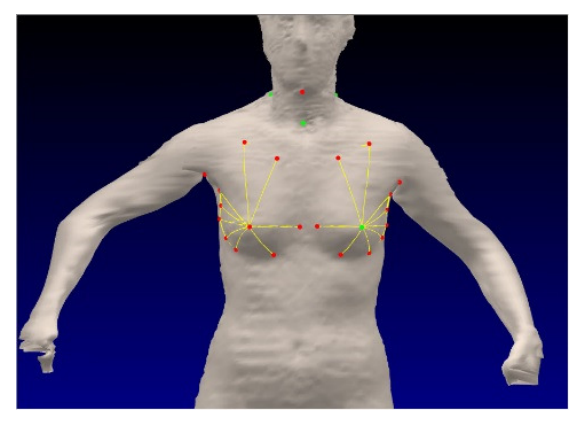

"Surface" Mode

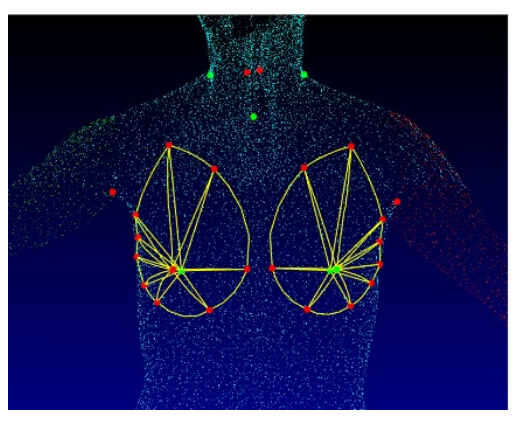

"Point Cloud" Mode

Figure 12. Breast Point Placement for "Breast Volume" Measurement Extraction Profile

One can readily see the difference between the "bust volume" measurement profile designed for the clothing industry, and the "breast volume" profile designed to meet the needs of the surgeon.

Figure 13 depicts the point-cloud image of the 3D body model in a supine position, with the head foremost in view. Note the distances between the points representing the apex of each nipple complex and its associated depth point. The depth points are meant to approximate the plane of the chest wall. The volume from each breast is calculated within the boundary of the points that outline each breast. The volume information is displayed in cubic centimeters (cc). The "base" surface area, that is, the area estimating the chest wall is displayed in centimeters squared.

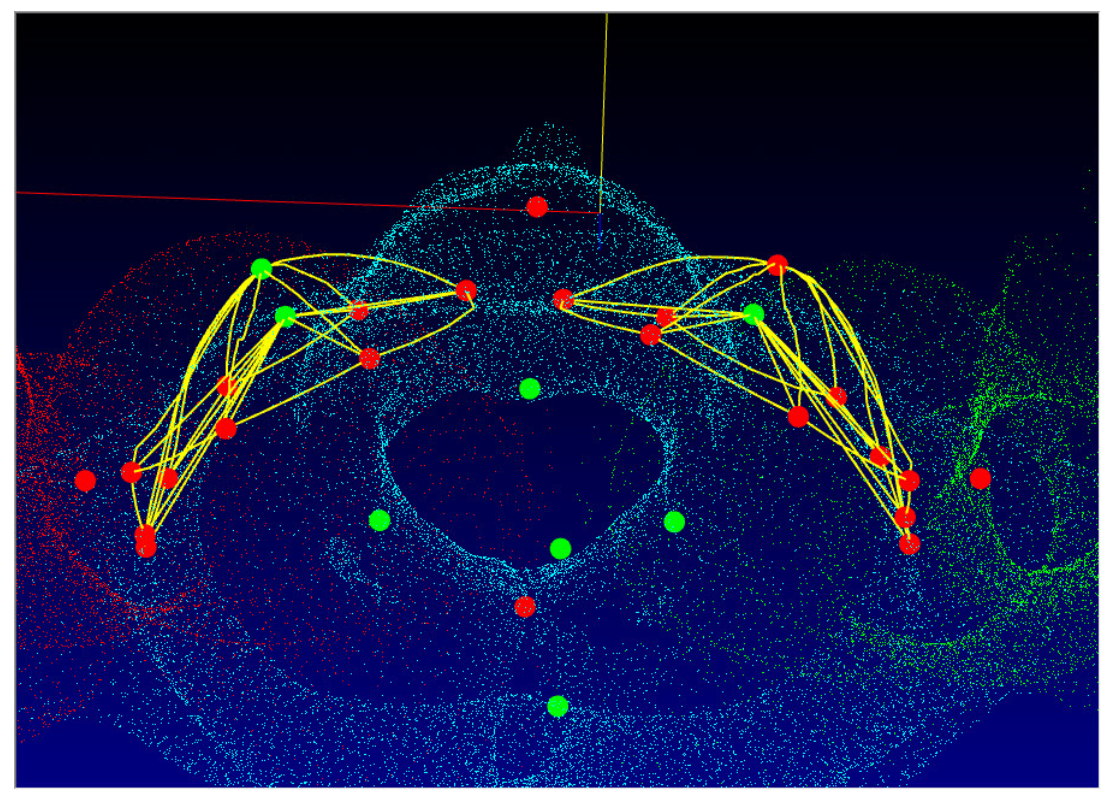

Figure 13. Supine Point Cloud View of Adjustable Breast Points and Breast Depth Points

In practice, it has been found that the volume information calculated is extremely sensitive to the location of the breast points. Moreover, consistent posture on the part of the patient during the scan acquisition is of the utmost importance. This technique for determining the breast volume has promise for breasts that do not have a high degree of ptosis. The length from the lowest point of the breast upward to the inframammary fold, in ptotic breast conditions, distorts volume calculations. 
Figure 14 presents the measurement points that the surgeon manually placed along the breast outlines of a post-operative breast augmentation patient, along with the extracted volume and surface area measurements. This is the same 3D body model as displayed in Figure 10.

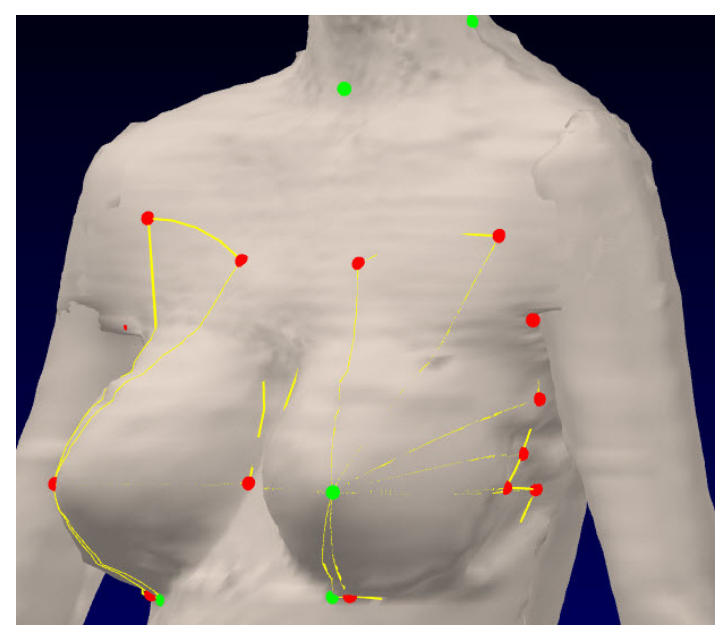

Display All Measurements Units : $\mathrm{cm}$

NV BustVolumeLeft : 812.9

NV_Bust_Volume_Right : 813.8

NV_BustBaseSurfaceleft : 291.1

NV_BustBaseSurfaceRight : 291.3

Figure 14. Volume and Surface Area Extraction from Applying the "Breast Volume" Template

The volume, in cc, for each breast as extracted by the programmable measurement extraction profile is quite similar

\section{Case 2. Breast Reduction}

Breast reduction surgery generally entails removal of a portion of each breast, reconstruction of the breast and relocation of the nipple complex. The desired outcome is a symmetrical breast appearance, proportional with the overall dimensions of the body.

Figure 15 presents an artist's rendering of a typical breast reduction procedure. In this example, the breasts appear to be symmetric, though breast reduction patients often present with an asymmetric condition.

There is a formulaic pattern, known as a "Wise Pattern" that is followed for the relocation of the nipple complex as part of the breast reduction procedure. Following this pattern, which is marked with pen on the pre-operative patient's chest area results in the symmetrical relocation of the nipple complex and common areola diameters. However, the use of this pattern does not determine the overall shape of the reduced breast. Currently, the surgeon must use his judgement, and the judgement of others present in the operating theatre to assess the overall symmetry of the surgically reduced breasts.

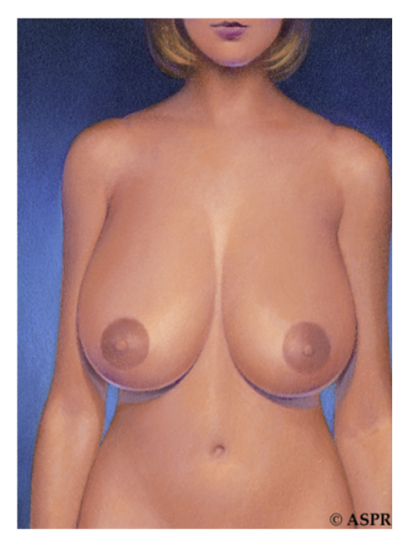

Presentation

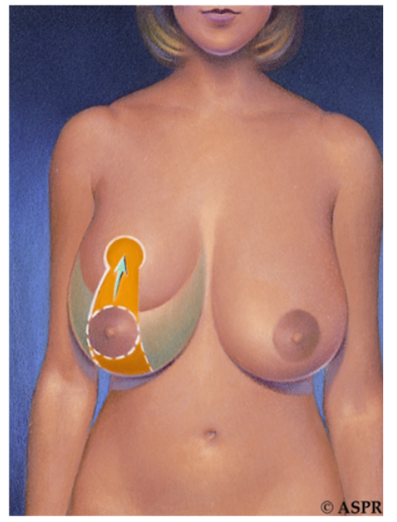

Relocation of Nipple Compex

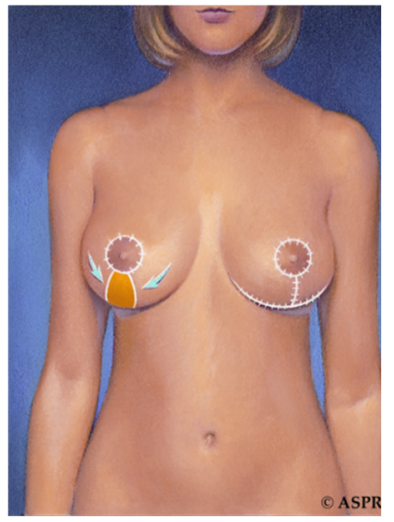

Removal of Breast Tissue

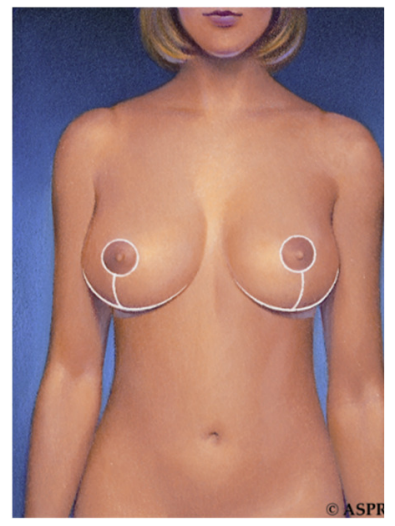

Closure

Figure 15. Artist's Rendering of the Steps Involved in a Breast Reduction Procedure

The application of the breast augmentation measurement profile discussed above can assist in "quantifying" an asymmetric breast condition for breast reduction patients. Due to the shape of the 
breasts, however, the measurements that result from the initial automatic measurement extraction are usually in error. Many of the landmarks and other programmable points require significant relocation. Once relocated, the breast augmentation template is reapplied to the modified $3 \mathrm{D}$ body model. The linear and contoured measurements that result from this extraction then appear to be accurate.

FIGURE 16 depicts pre-operative photographs of a patient that presented for a breast reduction. Underneath the photographs are equivalent images taken from the patient's preoperative 3D body model. The breast measurement template discussed above had been applied, and the yellow lines represent the measurement contours.

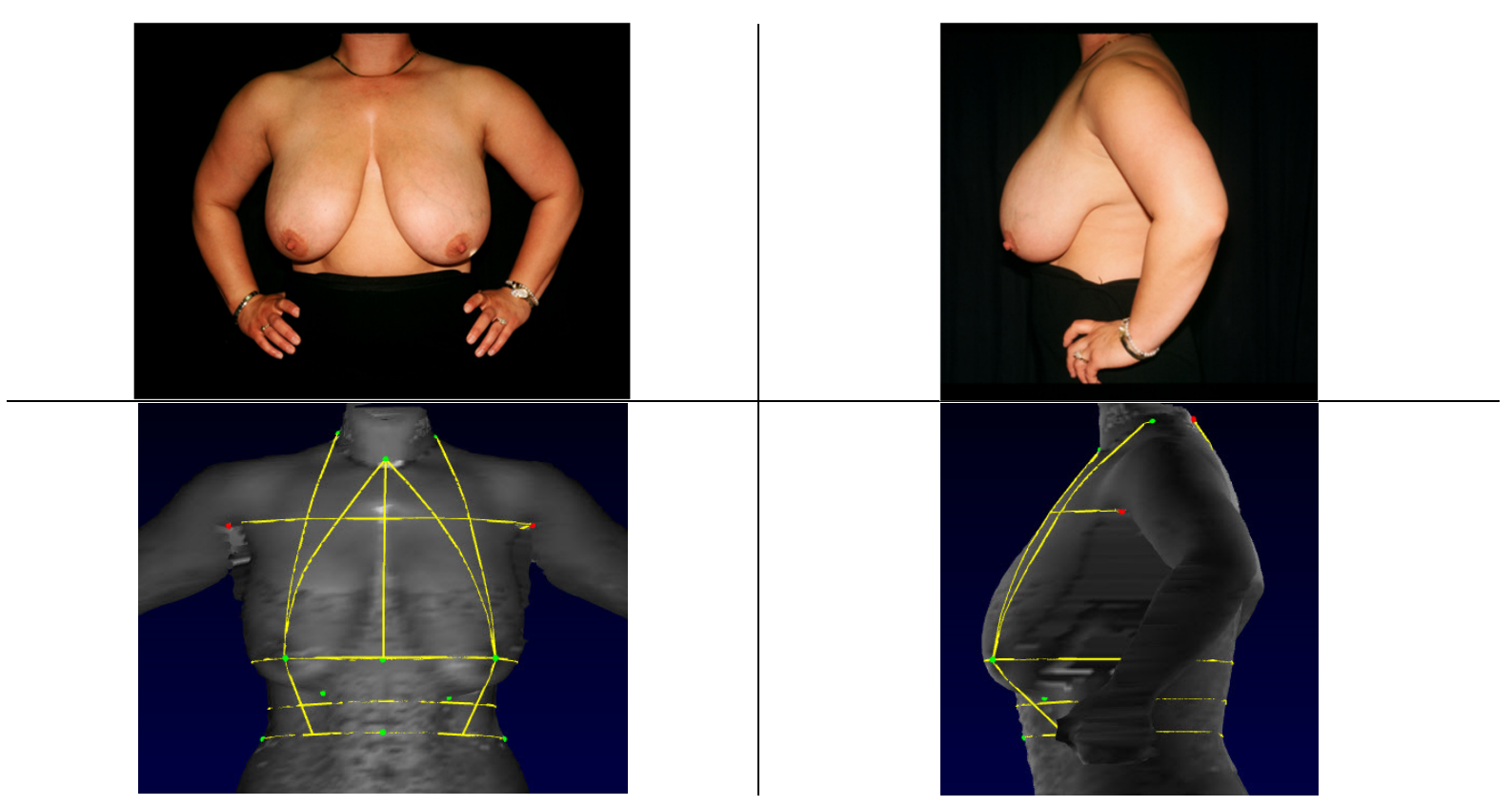

Figure 16. Photographs of Pre-Operative Breast Reduction Patient and Equivalent 3D Body Model

Figure 17 depicts the extracted measurements.

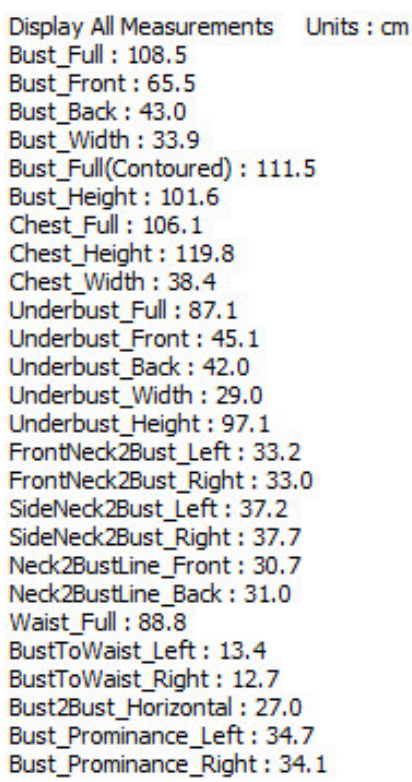

Figure 17. Measurements Extracted from the 3D Body Model of the Breast Reduction Patient

Observation of the "Bust_Prominance" and "FrontNeck2Bust" measurements displayed in Figure 17 implies that the pre-operative breasts are symmetrical. 
Figure 18 displays the results of the breast reduction procedure. Below the post-operative photographs are the equivalent images taken from the patient's post-operative 3D body model. Figure 19 compares the pre- and post-operative extracted measurements.

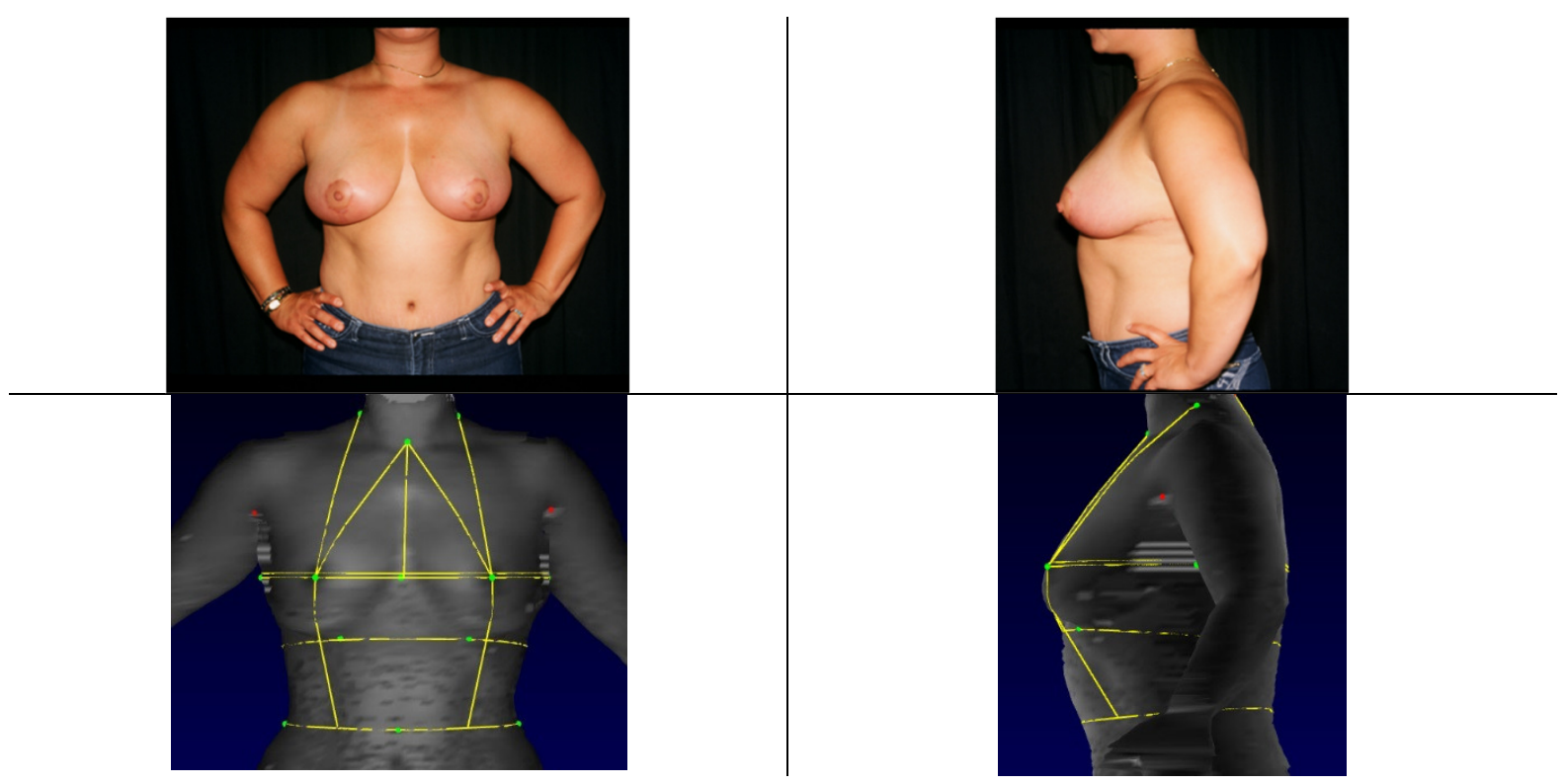

Figure 18. Post-Operative Photographs of Breast Reduction Patient and 3D Body Model Images

\begin{tabular}{|c|}
\hline $\begin{array}{l}\text { Display All Measurements Ur } \\
\text { Bust_Full : } 108.5 \\
\text { Bust_Front : } 65.5 \\
\text { Bust_Back : } 43.0 \\
\text { Bust_Width : } 33.9 \\
\text { Bust_Full(Contoured) : } 111.5 \\
\text { Bust_Height : } 101.6 \\
\text { Chest_Full : } 106.1 \\
\text { Chest_Height : } 119.8 \\
\text { Chest_Width : } 38.4 \\
\text { Underbust_Full : } 87.1 \\
\text { Underbust_Front : } 45.1 \\
\text { Underbust_Back : } 42.0 \\
\text { Underbust_Width : } 29.0 \\
\text { Underbust_Height : } 97.1 \\
\text { FrontNeck2Bust_Left : } 33.2 \\
\text { FrontNeck2Bust_Right : } 33.0 \\
\text { SideNeck2Bust_Left : } 37.2 \\
\text { SideNeck2Bust_Right : } 37.7 \\
\text { Neck2BustLine_Front : } 30.7 \\
\text { Neck2BustLine_Back : } 31.0 \\
\text { Waist_Full : } 88.8 \\
\text { BustToWaist_Left : } 13.4 \\
\text { BustToWaist_Right : } 12.7 \\
\text { Bust2Bust_Horizontal : } 27.0 \\
\text { Bust_Prominance_Left : } 34.7 \\
\text { Bust_Prominance_Right : } 34.1\end{array}$ \\
\hline
\end{tabular}

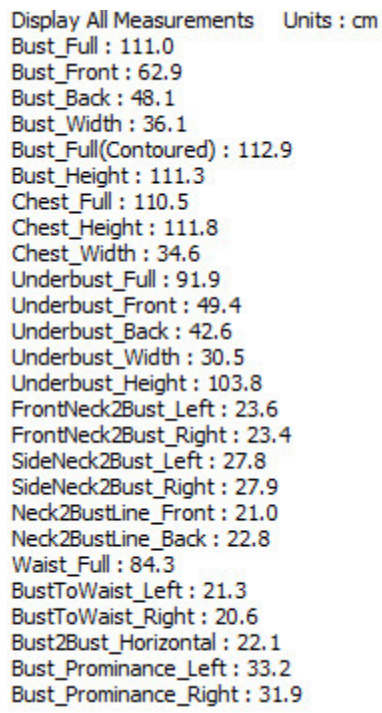

Figure 19. Pre- and Post-Operative Measurement Comparisons

Examination of the "Bust_Prominance" and "FrontNeck2Bust" measurements indicate that the postoperative breasts appear to be symmetric. This is visually apparent by viewing the "triangle" formed by the "FrontNeck2Bust" and "Bust2Bust" measurement lines.

There are two interesting observations to be made between the pre-operative and post-operative bust measurements presented in Figure 19.

The first observation is the difference between the pre-operative and post-operative Bust_Full measurement. The pre-operative measurement was $108 \mathrm{~cm}$. The post-operative measurement is 111 $\mathrm{cm}$. The pre-operative Bust_Height measurement was $101.6 \mathrm{~cm}$. This is the measurement from the height of the nipple complex, from the floor of the scanner. The post-operative Bust-Height measurement is $111.3 \mathrm{~cm}$. Collectively, these two measurements indicate that the result of the breast reduction procedure was to lift the profile of the breasts as well as increase the circumference of the bust, even though the breasts have been reduced in overall size. 
The second observation is that the "FrontNeck2Bust" measurements are close to the 23-cm template that was used to mark the relocation area for the nipple complex of each breast.

Depending on the degree of asymmetry, the size of each breast and possible ptotic condition, the breast volume measurement profile is of limited use for application on the pre-operative breast reduction patient. This is because the base of the breast is often well below the inframammary fold and the nipple complex with its associated "depth point" may not be able to be placed in position to accurately estimate the chest wall. It is a "judgment call" by the surgeon as whether to accept this pre-operative breast volume information as valid. Application of the breast volume template to the pre-operative breasts of this patient yielded volume results that the surgeon rejected.

Both measurement templates used for the breast augmentation procedure appear have value as a postoperative evaluation tool. Measuring the differences between the pre-operative linear, contoured and circumferential measurements with their associated post-operative measurements quantify the physical changes resulting from the breast reduction procedure. The breast volume measurement profile, with its movable points can yield an estimation of the post-operative volume for each breast. It is possible to estimate the volume of the pre-operative breast by weighing the tissue removed from each breast, in grams, and adding that number to the volume, in cc, as measured by the volume returned by applying the breast volume measurement extraction profile to the post-operative 3D body model.

\section{Summary of measurement templates created to assist breast augmentation and breast reduction procedures.}

The two templates used to evaluate pre-operative and post-operative breast augmentation procedures, the breast measurement template and the breast volume template, can be used for breast reduction surgery.

The first template consists of linear, contoured linear and circumferential measurements. Once the landmarks and movable points are properly located, the extracted measurements assist in quantifying an asymmetric condition. Key measurements include bust prominence, the distance from the suprasternal notch to each nipple, and the distance between the nipple complexes.

Asymmetries identified for breast reduction patients can be addressed and corrected during the surgical procedure. However, the surgeon understands that the breasts must be reduced individually. This is different from the case of breast augmentation, where implants of the same volume are generally used, and any existing asymmetries not identified and corrected would be magnified because of the augmentation procedure.

The second template, the breast volume template, requires the surgeon to locate the points that outline the definition of the breast as presented in Figures 11 and 12. This differs from traditional clothing measurement templates that attempt to define the bust for undergarment design. Once these points are moved into place, and the "breast depth point" has been situated, application of this template returns the left and right breast volume, in cc.

It has been noted that the volume of each breast, as defined by the location of the outline points and the depth point, is sensitive to the posture of the patient when the 3D body scan is acquired. It has also been noted that a ptotic breast condition affects the accuracy of the volume calculations.

\section{Case 3. Abdominoplasty}

Excepting the waist measurement, whose inclusion in an extraction profile is a requirement for the scanner software, the breast measurement and breast volume templates focused on the upper torso. Hips measurements were later added so that the patient and the surgeon could record traditional bustwaist-hips "proportions" before and after breast augmentation or breast reduction surgery. In the United States, the measurements were extracted in both centimeters (for the surgeon) and inches (for the patient).

Abdominoplasty, also known as a "tummy-tuck" addresses the abdominal area. Linear and contoured linear measurements offered by the scanner software for this area of the body appear to be of little use in this procedure, apart from a measurement known as the "CrotchLength_Full." The primary measurements of interest are circumferential in nature.

Figure 20 presents an artist's rendering of an abdominoplasty procedure. 

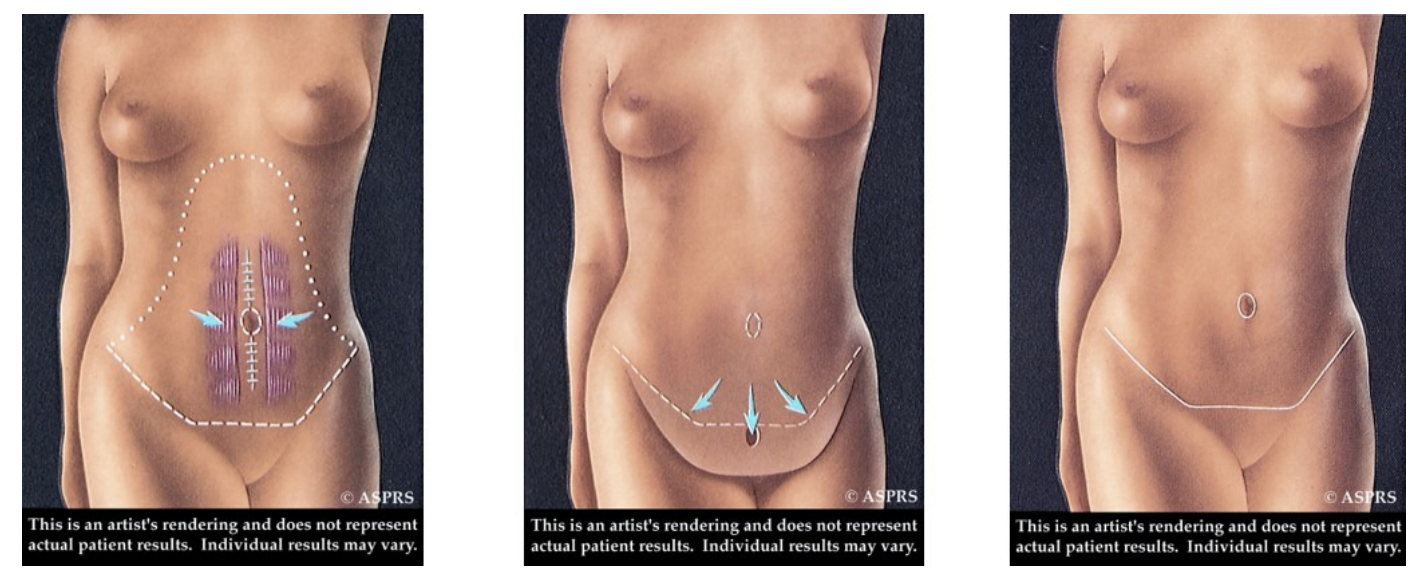

Figure 20. Areas of the Body Affected by an Abdominoplasty Procedure

Here, the surgeon is concerned with high lateral tension (upper dotted line pattern), rectus muscle plication (cross-hatched area) and relocation of the navel.

Figure 21 displays the body markings outlining a typical abdominoplasty procedure. This procedure aims to "lift and flatten" the abdominal area. In conjunction with the abdominoplasty procedure, liposuction is generally performed bilaterally on the "flanks" of the patient. The 3D body model images are shown in "color" display mode. These markings would not appear if the images were presented in "surface" or "point cloud" display modes.
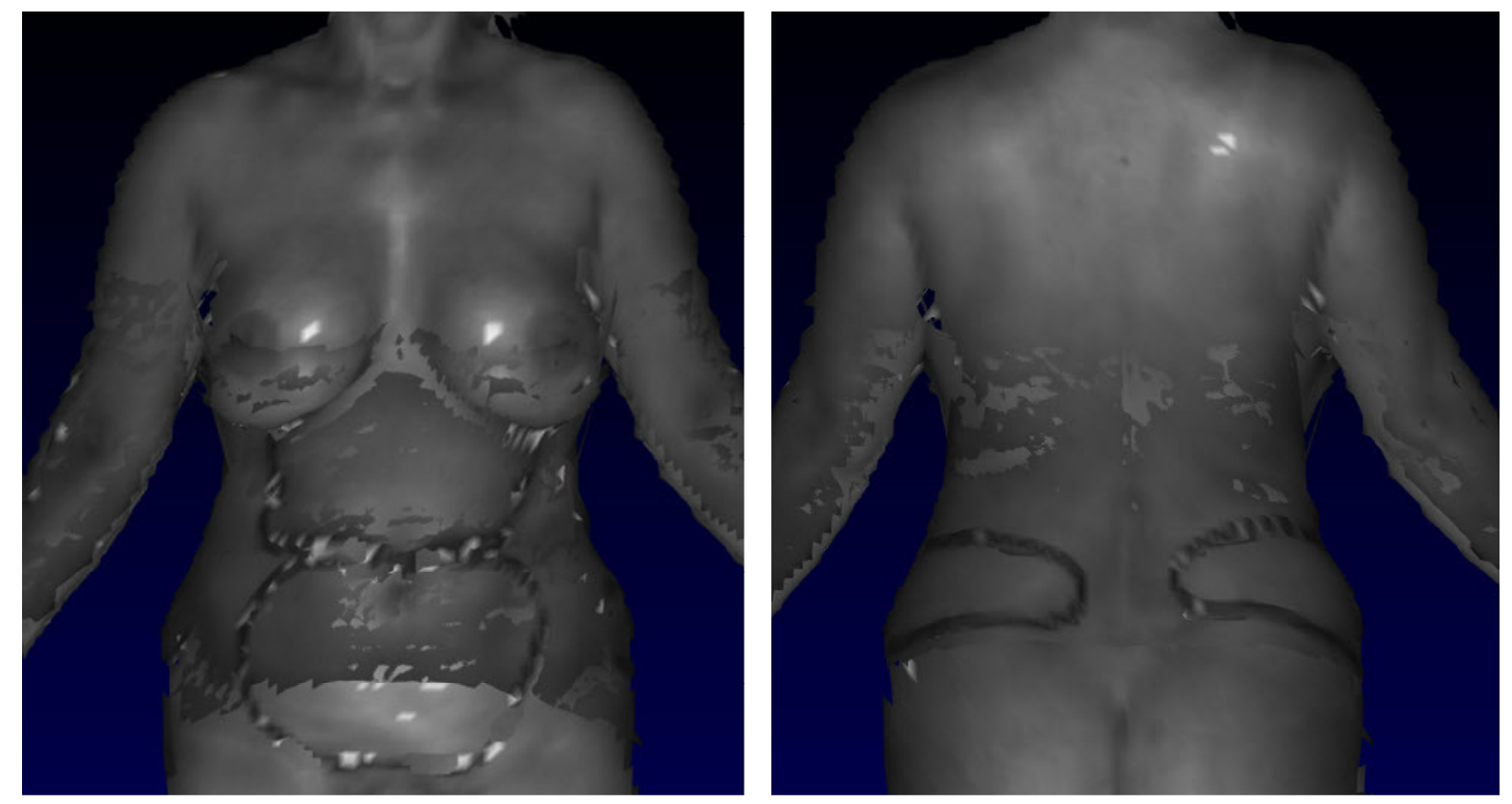

Figure 21. Body Markings Displayed on the 3D Body Model for a Pending Abdominoplasty Procedure

When the resected tissue is surgically closed, the effect is to tighten and lift the abdomen, slimming the waist, giving a trimmer appearance post-surgery. The areas targeted for liposuction are shown in the image on the right.

Primary measurements of interest for documenting the pre-operative abdominoplasty patient are waist, hips, stomach, abdomen, and seat circumferences.

Figure 22 displays the application of a lower body measurement template used to evaluate the 3D body model of a pre-operative abdominoplasty patient. The template has also been applied to the postoperative body model. The arms have been hidden by a display option within the scanner software program. 


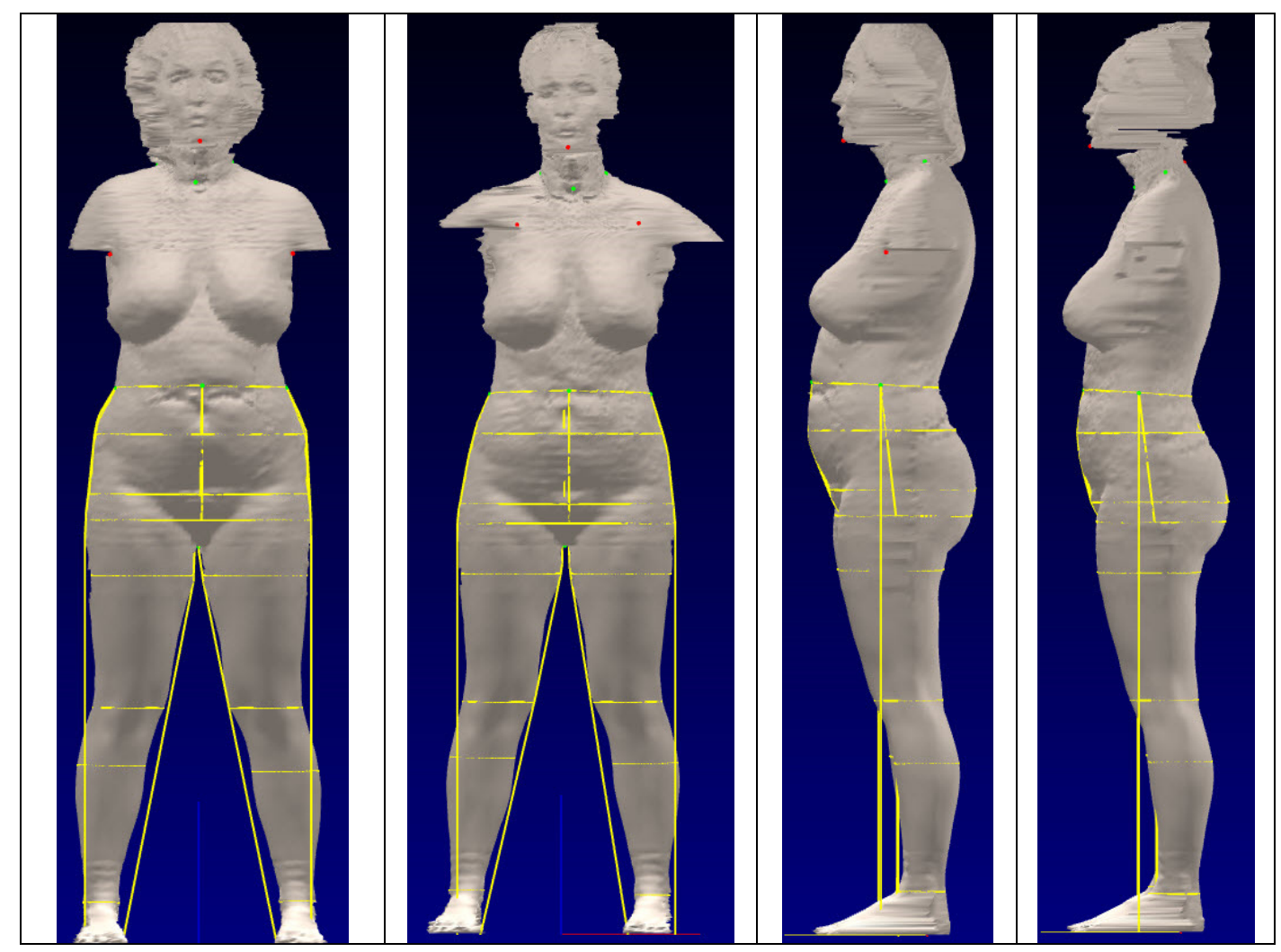

Figure 22. Application of the Lower Body Measurement Template for Abdominoplasty Procedure

Figure 23 presents the measurement changes between the pre- and post-operative 3D body model.

\begin{tabular}{|c|c|}
\hline $\begin{array}{l}\text { Display All Measurements } \\
\text { Waist_Full : } 83.8 \\
\text { Waist_Width : } 29.7 \\
\text { Hips_Full : } 106.6 \\
\text { Hips_Width : } 39.4 \\
\text { Seat_Full : } 104.8 \\
\text { Seat_Width : } 38.1 \\
\text { Seat_Back_Angle : } 24.5 \\
\text { Abdomen_Full : } 101.1 \\
\text { Abdomen_Width : } 37.0 \\
\text { Thigh_Left : } 57.3 \\
\text { Thigh_Right : } 58.4 \\
\text { Knee_Left : } 37.9 \\
\text { Knee_Right : } 37.6 \\
\text { Calf_Left : } 36.4 \\
\text { Calf_Right : } 36.9 \\
\text { Ankle_Girth_Left : } 22.8 \\
\text { Ankle_Girth_Right : } 22.9 \\
\text { Outseam_Left : } 97.7 \\
\text { Outseam_Right : } 98.0 \\
\text { Inseam_Left : } 70.7 \\
\text { Inseam_Right : } 70.6 \\
\text { CrotchLength_Full : } 71.6 \\
\text { Waist_to_Hips_Left : } 23.8 \\
\text { Waist_to_Hips_Right : } 24.0 \\
\text { Waist_to_Hips_Front : } 24.8 \\
\text { Waist_to_Hips_Back }: 23.4 \\
\text { Waist_Height_Front : } 97.3 \\
\text { Waist_Height_Back : } 96.4 \\
\text { Hips_Height : } 73.8 \\
\text { Seat_Height : } 78.3 \\
\text { Abdomen_Height : } 88.8 \\
\text { Waist_to_Seat_Left : } 19.3 \\
\text { Waist_to_Seat_Right : } 19.5 \\
\text { Waist_to_Seat_Front : } 19.7 \\
\text { Waist_to_Seat_Back : } 18.8\end{array}$ & Units : $\mathrm{cm}$ \\
\hline
\end{tabular}



Figure 23. Pre- and Post-Operative Measurement Extractions for Abdominoplasty Procedure

Post-operative circumferential measurements, such as waist, seat, abdomen, thigh show consistency with the reduction expected from the abdominoplasty procedure. 
Of some interest are the additional measurements provided by the scanner measurement software. The template included linear measurements, such as the width of the circumferential measurements, and the height of these measurements.

One can observe that the "Waist_Width," "Hips_Width," and "Abdomen_Width" indicate a corresponding physical reduction between the pre-operative 3D body model and the post-operative 3D body model.

\section{Post-surgical edema and its abatement}

Abdominoplasty with its adjunct liposuction results in significant edema post-procedure. 3D body scans of the post-operative patient at regular intervals assists in evaluating the cessation of edema.

Figure 24 displays a series of 3D body scans representing a patient who underwent an abdominoplasty procedure. This patient was scanned during each post-operative follow-up visit.

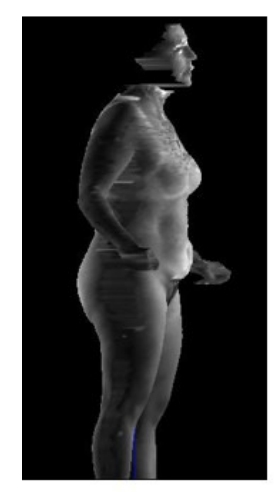

Pre-Op

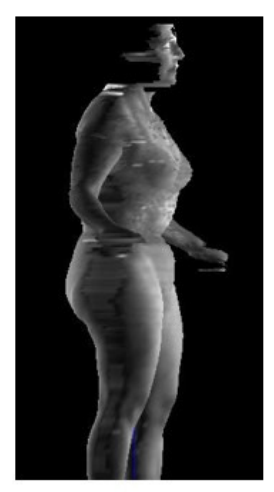

2 Month

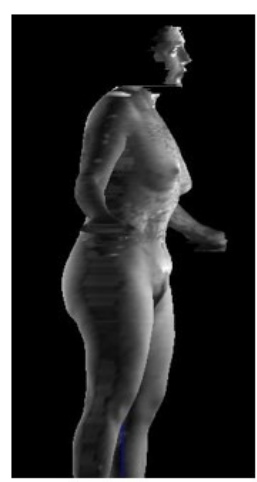

3 Month

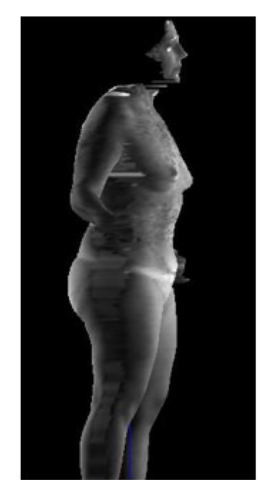

5 Month

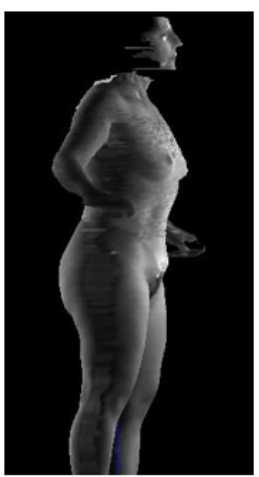

8 Month

Figure 24. A Series of Images from 3D Body Models of a Patient Who Underwent an Abdominoplasty Procedure

The lower body measurement template can be applied to extract measurements for each 3D body model. These measurement extractions can be exported using popular data formats, including several that can be imported directly into spreadsheet programs.

Measurement comparisons can be tabulated that compare measurement changes between successive scans, and overall measurement changes between the pre-operative 3D body model and the most recent 3D body model.

All 3D body models are placed into the patient's 3D digital archive. These 3D body models can be retrieved and measured using multiple measurement extraction templates. The 3D body models are unaffected by this process.

In our longitudinal analysis of the measurement changes for the 3D body models of the patient depicted in Figure 24, it was found that areas of the lower body, away from the area of surgery were affected in ways that would not be directly obvious. For instance, the knee circumference consistently measured larger post-surgery than the knee circumference measurement recorded on the pre-operative 3D body model.

\section{Case 4. Thigh Lift}

The thigh life procedure removes excess fat tissue from the inner thigh. To accomplish this, the surgeon uses an elongated " $T$ " or "U" pattern, depending on the course of action, starting where the inner thighs meet the buttocks. The vertical portion of the "T" or "U" extends downward to, and terminates above, the inner portion of the knee.

Figure 25 is an artist's rendering of the sections of the lower body that are affected by the thigh lift procedure. 


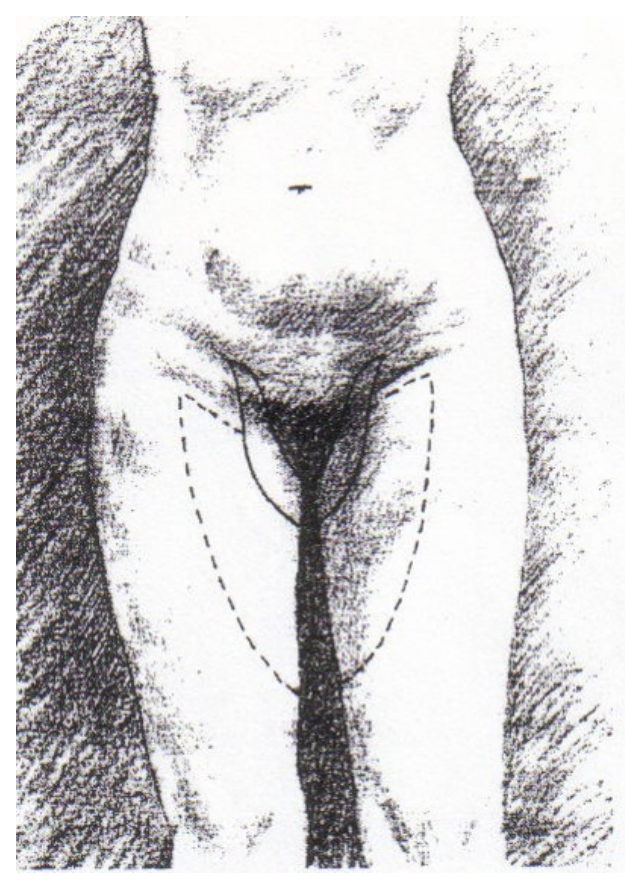

Figure 25. Artist's Rendering of Areas Affecting by Thigh Lift

The surgeon considers transverse groin incisions and vertical incisions along the medial thigh. Liposuction of the patient's flanks and upper thigh area often accompany this procedure.

Figure 26 displays the 3D body model of a patient that has been marked up in preparation for a thigh lift and liposuction procedure.
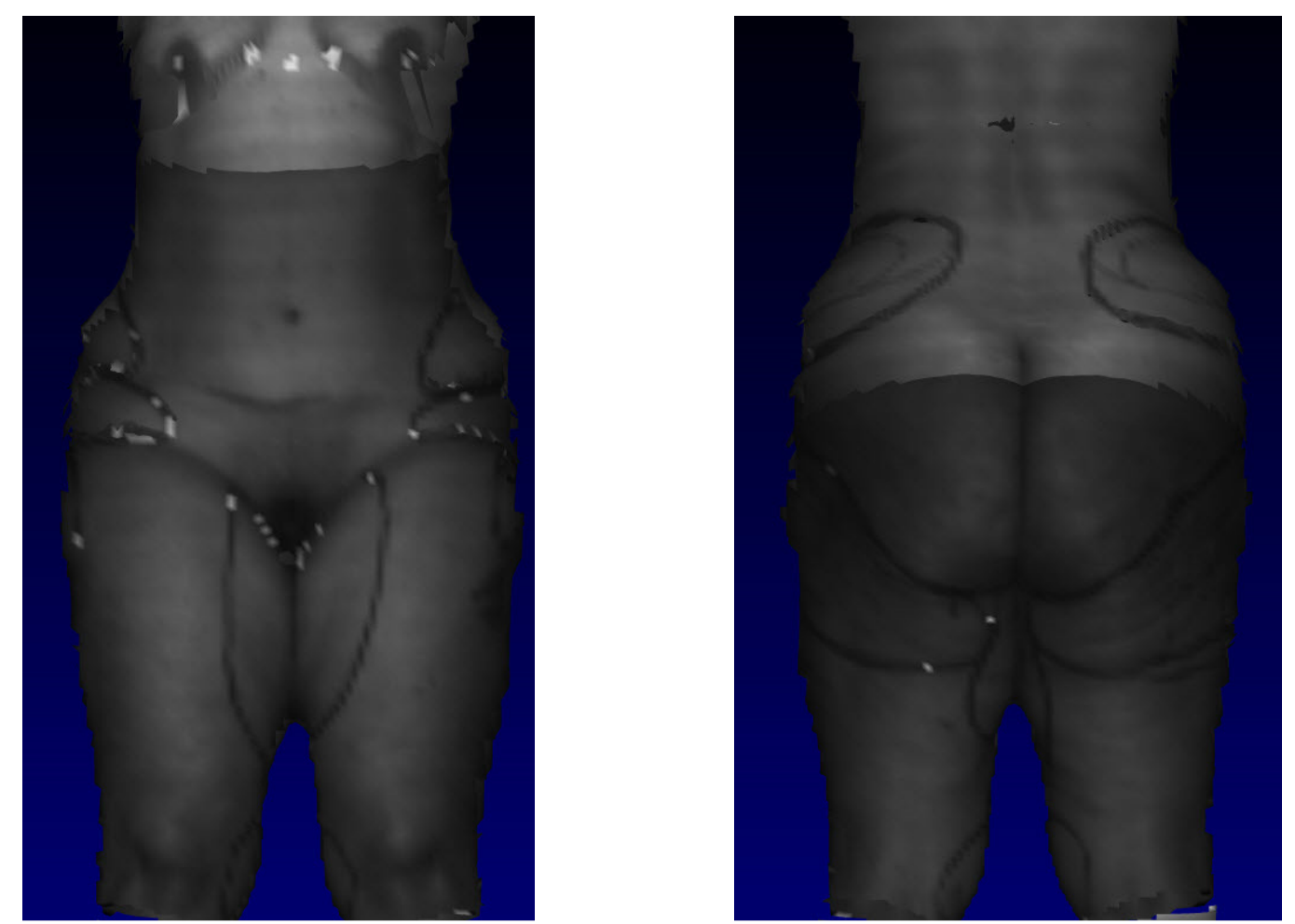

Figure 26. 3D Body Model Image of Pre-Operative Markings for Thigh Lift and Liposuction

One can observe the markings for the inner thigh. In addition, the markings on the patient flanks, below the buttocks and above the inner knee area indicate areas for liposuction. 
The resected areas of the thighs after tissue has been removed are then surgically rejoined. The result is reduction in each thigh circumference. The adjunct lipoplasty procedure affects the waist, hips, abdomen and seat circumferences. As with the other types of surgical procedures described above, longitudinal 3D body scanning and associated measurement extraction help evaluate the abatement of edema. The "true" result of the surgery can be validated when the edema has receded.

Figure 27 presents pre- and postoperative photographs of a patient who presented for a Radical Thigh Lift. No liposuction was performed as part of this surgery.

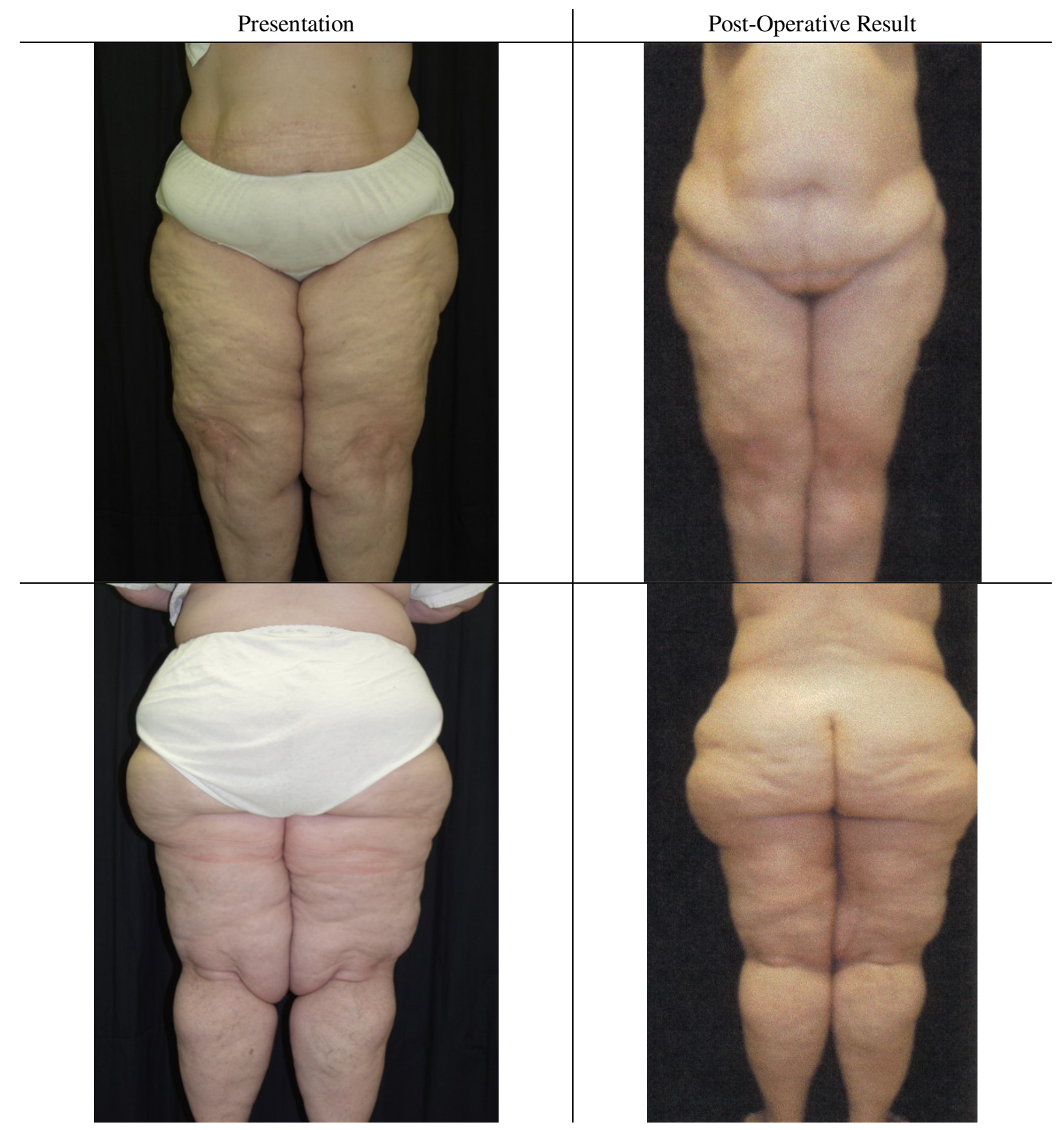

Figure 27. Pre- and Post-Operative Photographs of the Results of a Radical Thigh Lift

Note that these 2D photographs do not have a center of origin, as reflected by the different distances from which the photographs were taken.

Figure 28 displays the frontal and posterior images of the pre-operative and 10-week post-operative 3D body models. 


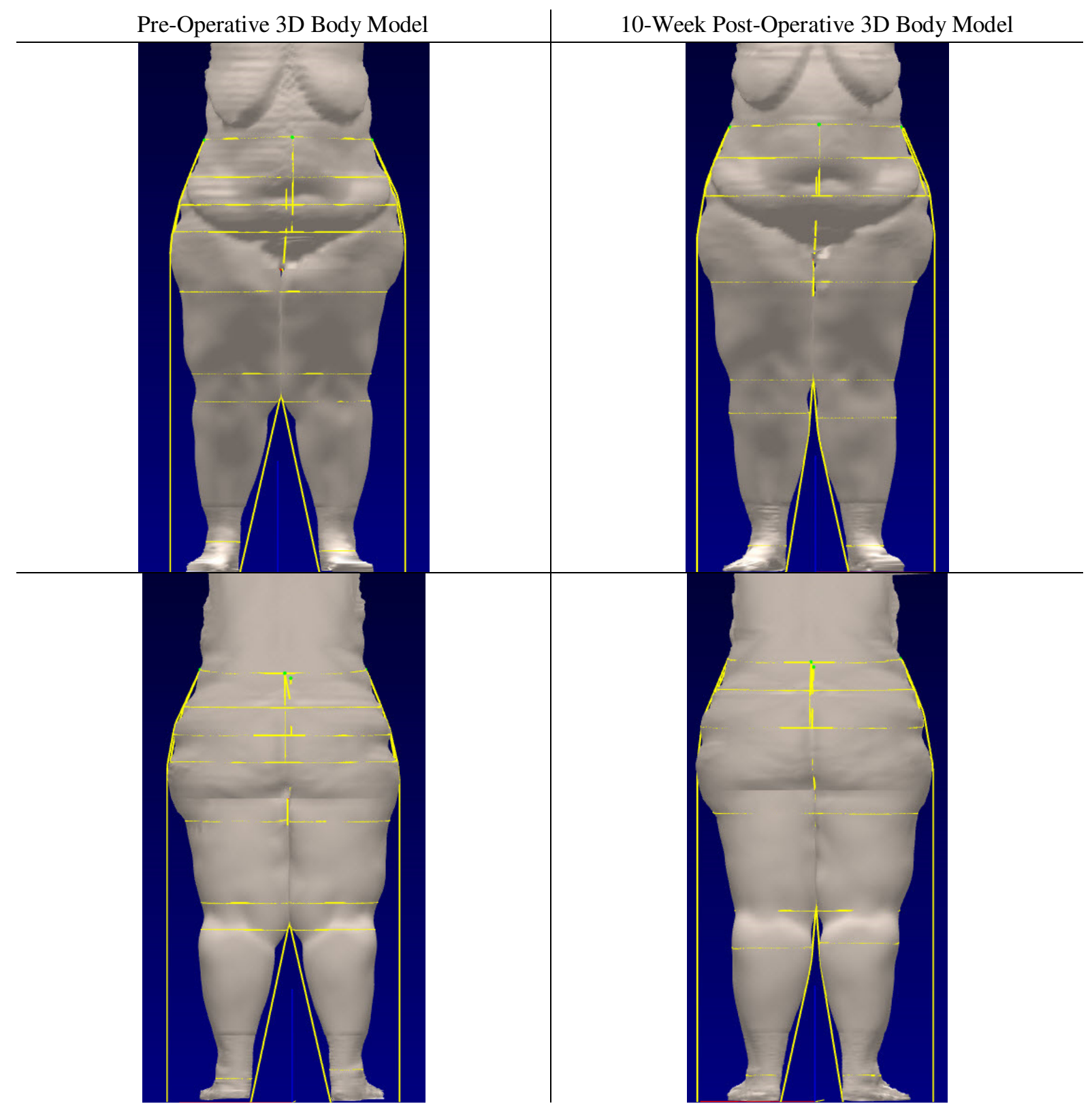

Figure 28. Pre- and 10-Week Post-Operative Images of the Patient's 3D Body Models

Figure 29 presents the tissue removed from each inner thigh. Note the distinct "T-pattern." The photograph on the left displays the area of the left thigh where the tissue was removed and the resulting scar.
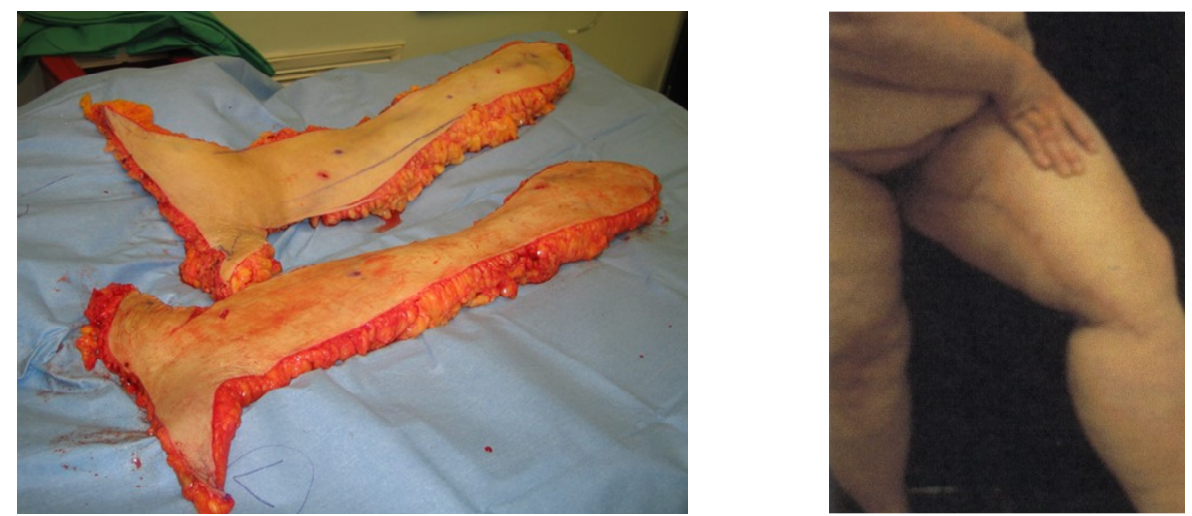

Figure 29. Tissue Removed from Inner Thighs and Post-Operative Scarring 
Figure 30 displays the pre-operative and post-operative measurements extracted by the lower body measurement template.

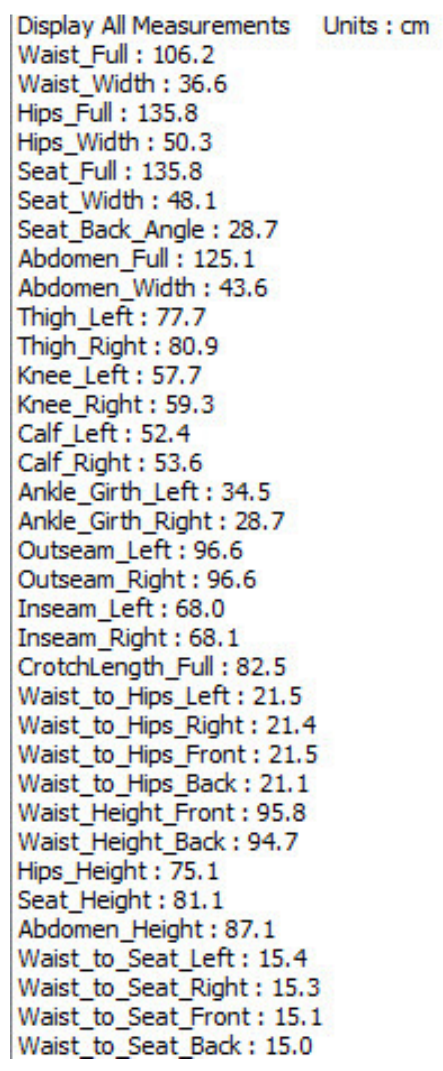

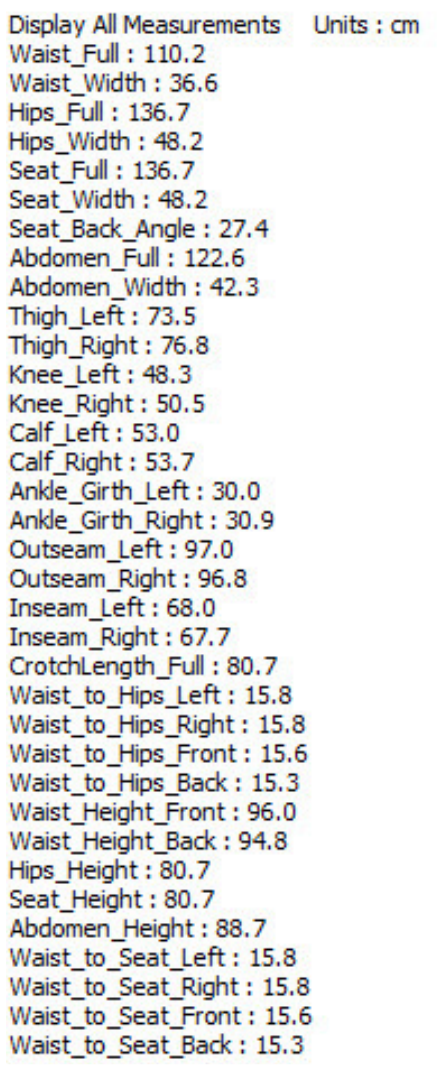

Figure 30. Lower Body Measurements Extracted from the Pre- and Post-Operative 3D Body Models

Curiously, the pre-operative right leg circumferential measurements, such as the thigh, knee and calf are greater than the similar measurements extracted for the left leg. Careful viewing of the pre-operative photographs or the pre-operative 3D body model indicate that this is the case. It is confirmed and quantified by the measurements extracted by the scanner software.

One can observe that the left and right thigh circumferential measurements have been reduced by over $4 \mathrm{~cm}$ each, and the left and right knee circumferences have been reduced by over $9 \mathrm{~cm}$ each. These were the areas affected by the removal of tissue. The patient's post-operative waist and hips measurement appear to have increased between the 10 weeks that had elapsed from the date of the surgical procedure.

The differences between the pre-operative right and left thigh circumferences was $3.2 \mathrm{~cm}$. This might have been taken into consideration prior to the markings on the thighs for tissue removal. However, the surgeon felt that a symmetrical T-pattern on each inner thigh was the conservative approach.

\section{Summary and conclusions}

The use of a 3D body scanner and its associated measurement software measurement capabilities requires the surgeon to adopt unfamiliar technology. Viewing the patient's 3D body model literally adds a new dimension to the consultation and evaluation process. Measurements specific to the body "area of interest" can be selected from the many measurements available within the scanner measurement software. Many of these measurements are related to the garment industry, but there are some measurements that are directly suitable as an aid for pre-operative body contour evaluation.

New measurements can also be created by utilizing the simple programming capabilities of the scanner measurement editor. Combinations of measurements can be defined and then acted upon by mathematical operations such as addition, multiplication, subtraction and division of elemental measurements. 
It is quite possible that new measurements will be invented that could redefine how existing body contours are evaluated. It is also possible to create measurement templates that have far too much measurement information, with most measurements being meaningless to the surgeon.

The Breast Measurement Template, the Breast Volume Template and the Lower Body Measurement Template presented in this paper are the direct results of trial, error and adjustment, based on experience. They are far from complete. However, the measurements they extract from specific areas of the body have proven to be helpful to the surgeon for evaluating pre-operative body contours. This assists in the planning of the surgical procedure.

Applying the same measurement template to the post-operative 3D body model documents physical changes resulting from the specific surgery. This validates the surgical results.

\section{A note on 3D digital archiving}

Many of the case examples discussed in this paper took place between 2002 and 2005. This paper was written in 2017. The 3D body models acquired during those years can be measured with measurement software that is current at the time of this writing.

\section{References for further reading}

Integrating a 3D Body Scanner into an Active Bariatric Surgery Clinic: Practical Experiences, History, Tips and Pitfalls. David Stefan, M.S.E.E., David A. Gilbert, M.D., Stephen D. Wohlgemuth, M.D. Paper ID 562014 3D Body Scanning Conference. Lugano, CH. October 2014.

This paper details the history of the $3 D$ whole-body scanners employed by The Hague Center for Cosmetic Surgery. It includes a discussion of the many issues that needed to be resolved before 3D scanning of patients became integrated into the workflow of the clinic's personnel. There is a discussion of best practice protocols that are in use for the 3D scanner in a clinical setting.

Breast Augmentation Virtual Surgery Using 3D Body Scanning: Bridging the Gap between Patient Expectations and Surgical Practicalities. David A. Gilbert, M.D. Paper ID 552013 3D Body Scanning Technologies, Long Beach, CA, USA. November 2013.

This paper details the early efforts to develop an effective measurement template for use on breast augmentation and breast reduction patients. It also gives insight into the ability of the scanner to export $3 D$ body models to CAD programs, and how it is possible to perform "virtual surgery" to predict the outcome of breast augmentation and breast reduction procedures prior to conducting the surgery. 